\title{
Catalizadores magnéticos basados en Óxidos de Hierro: Síntesis, Propiedades y Aplicaciones
}

\author{
Magnetic catalysts based on Iron Oxides: \\ Synthesis, Properties and Applications
}

Angélica M. Escobaro ${ }^{a^{*}}$

Luis R. Pizzioa*

Gustavo P. Romanelli*

Fecha de Recepción: 03.06.2018

Fecha de Aceptación:12.12.2018

DOI: https://doi.org/10.19053/01217488.v10.n1.2019.88111

\begin{abstract}
Resumen
Esta revisión se centra en las propiedades magnéticas, estructura, síntesis y aplicación como catalizadores de las nanopartículas magnéticas de óxidos de hierro. En los últimos años son muchos los avances logrados respecto a su síntesis, control del tamaño y forma, donde se destacan métodos como la co-precipitación, microemulsión, síntesis hidrotermal y solvotermal, entre muchas otras. Pero debido a que las nanoparticulas de óxidos de hierro son fácilmente oxidables por el medio ambiente y por medios ácidos, se ve la necesidad de recubrirlas o protegerlas con materiales no magnéticos que en muchos casos funcionan también como sustancias funcionalizadoras a las cuales se une la fase activa. Así, es amplio el abanico de posibilidades de sustancias y compuestos protectores, tales como óxidos inorgánicos, polímeros y metales, al igual las técnicas para su funcionalización. Las nanopartículas magnéticas una vez funcionalizadas pueden usarse como bloques de construcción para la fabricación de una gran variedad de sistemas catalíticos, y en este trabajo se revisará brevemente algunos de estos sistemas y su aplicación en diferentes procesos como reacciones de Síntesis Orgánica, fotocatálisis y eliminación de metales y otras sustancias en aguas residuales, por citar algunos ejemplos. Finalmente, se delinearán algunas tendencias y perspectivas futuras en estas áreas de investigación.
\end{abstract}

Palabras claves: Nanopartículas magnéticas, óxidos de hierro, catálisis heterogénea.

a Centro de Investigación y Desarrollo en Ciencias Aplicadas “Dr. Jorge J. Ronco" (CINDECA), Departamento de Química, Facultad de Ciencias Exactas, UNLP-CCT La Plata, CONICET, 47 No 257, B1900AJK La Plata, Argentina.

* Correo electrónico: gpr@quimica.unlp.edu.ar (G.P.Romanelli) 


\begin{abstract}
This review focuses on the magnetic properties, structure, synthesis and application as catalysts of magnetic nanoparticles of iron oxides. In recent years there have been many advances in its synthesis, control of size and shape, which highlights methods such as co-precipitation, microemulsion, hydrothermal and solvothermal synthesis, among many others. But because the nanoparticles of iron oxides are easily oxidizable by the environment and by acidic means, it is necessary to coat them or protect them with non-magnetic materials that in many cases also function as functionalizing substances to which the phase is attached active Thus, the range of possibilities for protective substances and compounds, such as inorganic oxides, polymers and metals, is broad, as are the techniques for their functionalization. Magnetic nanoparticles, once functionalized, can be used as building blocks for the manufacture of a wide variety of catalytic systems, and in this work we will briefly review some of these systems and their application in different processes such as Organic Synthesis reactions, photocatalysis and elimination of metals and other substances in wastewater, to name a few examples. Finally, some trends and future perspectives in these research areas will be delineated.
\end{abstract}

Keywords: Magnetic nanoparticles, iron oxides, heterogeneous catalysis.

\section{INTRODUCCIÓN}

En las últimas décadas, la síntesis de nanopartículas magnéticas (NPMs) de óxidos de hierro se ha desarrollado intensamente, no solo por su interés científico fundamental, sino también por sus múltiples aplicaciones tecnológicas, dentro de las cuales se destacan: medios de almacenamiento magnéticos tanto analógicos como digitales [1], aplicaciones de biosensores [2], dispositivos médicos como agentes de contraste de Resonancia Magnética por Imágenes (RMI), terapia magnética y administración controlada de fármacos [3, 4], aplicaciones medio ambientales como remediación y tratamientos de agua mediante eliminación de contaminantes $[5,6]$, en la ciencia de los materiales [7] y en nanocatálisis [8-10], entre otros.

Centrándonos en el campo de la catálisis, los procesos a gran escala con catálisis heterogénea a menudo requieren que la separación y recuperación del catalizador, del medio de reacción, sea muy eficiente, tanto para minimizar la pérdida del catalizador, como para evitar la contaminación de los productos y reducir costos de producción. En el caso de partículas grandes de catalizador, normalmente se pueden retener en reactores o ser separados de los productos por filtración convencional o por centrifugación, pero cuando son nanomateriales, la recuperación utilizando los métodos convencionales es ineficiente y costosa, lo que impide su uso en la industria. Esto hizo que las NPMs sean tan interesantes para ser usadas en el capo de la catálisis, ya que su alto valor de magnetización, permite controlar de forma reversible su dispersión en el medio de reacción mediante la aplicación de un campo magnético, solucionando los problemas de separación y recuperación [11]. Así, la separación magnética de estos materiales, aunado a otras propiedades fisicoquímicas como su gran área superficial, baja toxicidad, facilidad de síntesis y viabilidad económica, hace que sea un procedimiento simple, limpio, ambientalmente amigable, económico y prometedor para aplicaciones tanto en la industria química como farmacéutica [12].

Por otra parte, en el rango de tamaños entre 1 y $100 \mathrm{~nm}$, en el cual se definen los materiales nanométricos, se producen fenómenos únicos que alteran sus propiedades físicas y químicas y los habilitan para nuevas aplicaciones. Dichos fenómenos dependen fuertemente del tamaño y morfología de las nanopartículas. Un ejemplo de ello es que cuanto más pequeña es una nanopartícula, mayor es su área superficial relativa, y propiedades como la conductividad eléctrica y mecánica cambian, pero lo más interesante, es que cada partícula se comporta como un monodominio magnético, presentando un fenómeno tan inusual como es el superparamagnetismo [13]. Lo anteriormente mencionado ha hecho que en la actualidad un gran número de investigaciones se centran en desarrollar métodos simples y efectivos para la síntesis de NPMs de forma controlada.

Aunque el interés por las NPMs como soportes de catalizadores ha ganado importancia en los 
últimos años, su uso se remonta a la década de 1970, cuando P.J. Robinson y colaboradores reportaron la síntesis de biocatalizadores enzimáticos, soportadas sobre precipitados de óxido de hierro recubiertos con amino-silano y celulosa [14]. En este estudio, las enzimas inmovilizadas en el soporte magnético mostraron una actividad catalítica constante independientemente de si el soporte se había magnetizado o no. Posteriormente, en la misma década, C. L. Hill y col [15] prepararon catalizadores de paladio soportado sobre carbono, sílica o alúmina con bajas cargas de magnetita, sintetizados mediante la deposición de una suspensión preformada de magnetita sobre los soportes y estudiaron una forma eficiente de separar los catalizadores mediante la técnica que denominaron separación magnética de alto gradiente (HGMS por sus siglas en inglés), logrando una separación completa $(<95 \%)$. La actividad del catalizador se estudió en la reacción de hidrogenación del ciclohexeno con un rendimiento del $80 \%$ y el rendimiento de la recuperación del catalizador se mantuvo constante durante 4 ciclos de reacción.

Desde entonces y hasta la actualidad, numerosos estudios se vienen realizando en el campo de los catalizadores magnéticos y aunque las sustancias magnéticas más utilizadas como soporte en sistemas nanocatalíticos han sido las nanopartículas superparamagnéticas de óxido de hierro, otros metales y aleaciones, como $\mathrm{Ni}$, Co, FePt y FePd, también se han utilizado como soporte magnético en estudios recientes [16].

Como desventaja, en general, las nanopartículas tienen una inclinación a agregarse para reducir su energía de superficie debido a su gran área superficial, y en el caso de las NPMs, la interacción dipolar magnética, junto con las interacciones de Van der Waals, aumenta las posibilidades de aglomeración de las partículas [17]. Estas interacciones entre las NPMs se pueden suprimir recubriendo las nanopartículas con una matriz no magnética orgánica/inorgánica o con surfactantes adecuados como los ácidos grasos. Dicha modificación minimiza la aglomeración al suprimir o reducir el efecto de Van der Waals, así como las interacciones magnéticas entre las nanopartículas. Estos recubrimientos, también protegen a las NPMs de oxidarse por el oxígeno del ambiente o de descomponerse en medios ácidos y adicionalmente, funciona como puntos de anclaje, permitiendo introducir especies catalíticas [18].

Hasta ahora se han diseñado un gran número de catalizadores magnéticos que involucran recubrimientos con polímeros [19], sustancias organometálicas [20] y surfactantes [21] y variadas especies activas como metales [22], enzimas [23] y ácidos orgánicos/inorgánicos [24]. Así mismo, se han estudiado diferentes métodos para inmovilizar el catalizador considerando las características químicas de cada especie. Por ejemplo, mientras que los catalizadores organometálicos y orgánicos normalmente se inmovilizan en una superficie de nanopartículas utilizando un protocolo de conjugación con un reactivo de reticulación, el método de impregnación se utiliza frecuentemente para especies metálicas, y en el caso de los biocatalizadores, el principal inconveniente es lograr que el sitio activo de las enzimas no quede oculto, o que pierda su estructura terciaria, por lo que se estudian métodos de inmovilización que permitan mantener su actividad y selectividad [25]

La gran variedad de catalizadores magnéticos que se pueden obtener variando el material de recubrimiento y la fase activa, permite que sea amplia la gama de reacciones orgánicas en los que se puedan utilizar, esto incluye reacciones de acoplamiento C-C, hidrogenación, oxidación, condensación aldólica y polimerización. La mayoría de las evaluaciones se realizaron con el foco en la reciclabilidad de los catalizadores. Además, en muchos casos, los catalizadores magnéticos se recuperaron mediante un simple proceso de separación magnética y las reacciones se repitieron con éxito sin ninguna pérdida de actividad catalítica [26].

En esta revisión, resumimos las rutas químicas para la síntesis de nanopartículas de óxido de hierro superparamagnéticas, los diferentes materiales de recubrimiento, la variedad de fases activas soportadas y las principales aplicaciones catalíticas. 
2. PROPIEDADES MAGNÉTICAS DE LAS NANOPARTÍCULAS

Existen cinco tipos de respuestas a un campo magnético aplicado según las cuales se pueden clasificar todos los materiales, estos son: diamagnetismo, paramagnetismo, ferromagnetismo, antiferromagnetismo y ferrimagnetismo (Figura 1) [27].

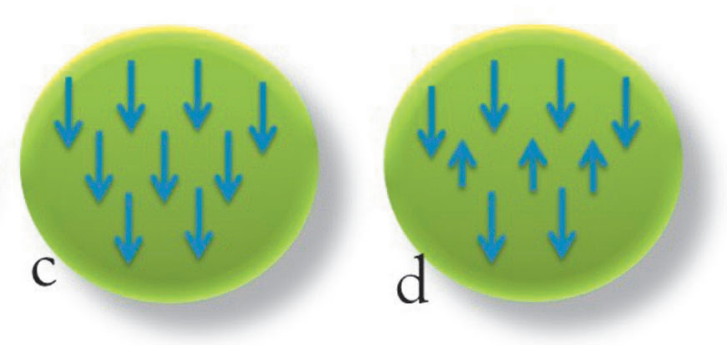

Figura 1. Diferentes tipos de comportamiento magnético. a) Paramagnetismo, b) Antiferromagnetismo, c) Ferromagnetismo y d) Ferrimagnetismo (Imagen adaptada de Daliya S. Mathew y col [27]).

En términos de la configuración electrónica, los materiales clasificados como ferromagnéticos, alinean todos sus momentos magnéticos en la misma dirección y sentido al aplicarles un campo magnético. Esto ocurre con los elementos como $\mathrm{Fe}$, Ni y Co. Además, esta alineación puede conferir una espontánea magnetización en ausencia de un campo magnético aplicado.

De igual forma, los materiales clasificados como ferrimagnéticos son aquellos cuyos átomos o iones tienden a asumir una posición ordenada en la misma dirección, pero no en el mismo sentido, de modo que se anulan entre sí parcial o completamente, sin embargo, al estar distribuidos de forma aleatoria y al no ser equivalentes, la magnetización no llega a ser cero, presentando así, al igual que los materiales ferromagnéticos, magnetizaciones permanentes y punto de saturación, aunque con valores menores. Los ejemplos más importantes dentro de esta clasificación son los óxidos de hierro como la magnetita $\mathrm{Fe}_{3} \mathrm{O}_{4}$. Por encima de una determinada temperatura (temperatura de Curie, $\mathrm{T}_{\mathrm{C}}$, en los ferromagnéticos y ferrimagnéticos,), se vencen las fuerzas de acoplamiento de los momentos magnéticos y se comportan como materiales paramagnéticos, con los momentos magnéticos desalineados [27].

Los materiales ferromagnéticos y ferrimagnéticos están divididos en dominios magnéticos, los cuales fueron postulados por primera vez por Pierre Weiss en 1907 [28] y que se refieren a regiones que engloban momentos magnéticos con una misma orientación, esto significa que los momentos magnéticos de los átomos individuales están alineados uno con el otro y que apuntan en la misma dirección. Dichas regiones están separadas unas de otras por paredes de dominio (Figura 2). En el caso de materiales nanométricos, las partículas de los materiales magnéticos actúan como monodominios dando lugar al efecto conocido como superparamagnetismo, donde cada partícula se comporta como un átomo paramagnético, pero con un gigantesco momento magnético, ya que todavía hay un orden magnético bien definido en cada nanopartícula [29].

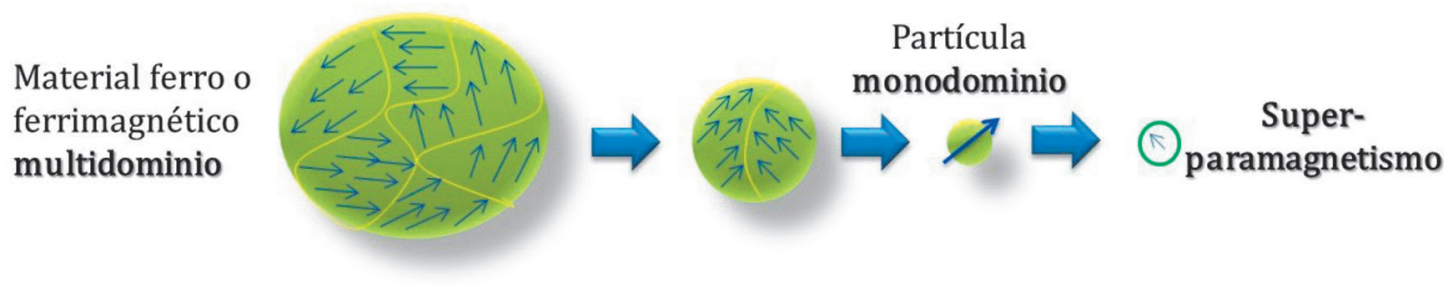

Figura 2. Esquema de los multidominios magnéticos según el tamaño de la partícula del material magnético. Imagen adaptada de R. Pujales [30]. 
En ausencia de un campo magnético, los diferentes dominios están alineados aleatoriamente, tendiendo a anularse entre sí. Como se muestra en la Figura 3.a) la separación entre dos dominios no es abrupta ya que energéticamente no es favorable, sino que la transición está formada por las paredes de dominios, donde la magnetización va girando progresivamente. $\mathrm{Al}$ aplicar un campo magnético (Figura 3.b y c), los momentos magnéticos rotan gradualmente, haciendo que las paredes de dominio se muevan y se produzca un crecimiento de uno de los dominios a expensas de sus vecinos.

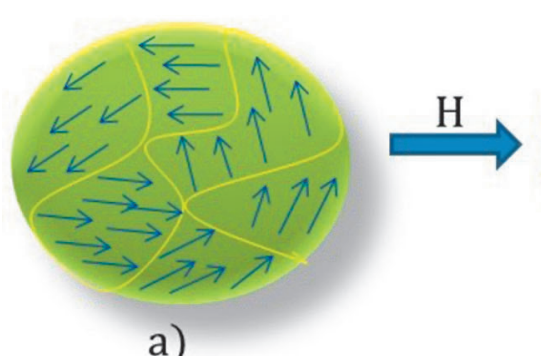

a)

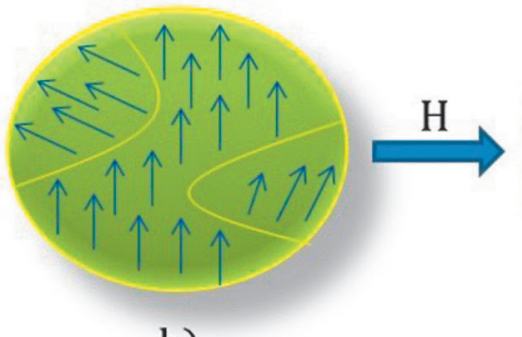

b)

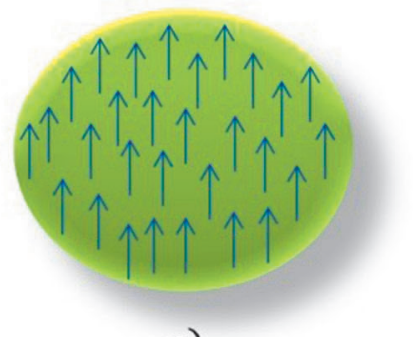

c)

Figura 3. Representación del movimiento de la pared de dominio bajo la aplicación de un campo magnético externo (H). a) Esquema de un material separado en multidominios magnéticos; (b) a campo distinto de cero, se desplazan las paredes y los dominios se empiezan a orientar paralelos al campo aplicado y (c) rotación de los dominios hasta orientar totalmente su magnetización paralela al campo aplicado. Imagen adaptada de M. Castellanos [31].

\subsection{Estructura y propiedades de los óxidos de hierro}

Como se ha mencionado, en la actualidad, las NPMs más estudiadas son las correspondientes a óxidos de hierro, las cuales contienen únicamente hierro como catión metálico y se destacan tres tipos, magnetita $\left[\mathrm{Fe}_{3} \mathrm{O}_{4}\right]$, maghemita $\left[\gamma-\mathrm{Fe}_{2} \mathrm{O}_{3}\right]$ y hematita $\left[\alpha-\mathrm{Fe}_{2} \mathrm{O}_{3}\right]$.

La hematita [32] es el óxido de hierro más estable y un semiconductor tipo $n$ en condiciones ambientales, donde la banda de conducción está compuesta por orbitales $d$ de $\mathrm{Fe}^{3+}$ y la banda de valencia (VB) por orbitales $3 d \mathrm{de} \mathrm{Fe}^{3+}$ con algunas mezclas de los orbitales $2 p$ del $\mathrm{O}$ no vinculante. Como se muestra en la Figura 4.a, presenta una estructura romboédrica donde los iones $\mathrm{Fe}^{3+}$ ocupan dos tercios de los sitios octaédricos que están confinados por la rejilla hexagonal. Es ampliamente utilizado en catalizadores, pigmentos y sensores de gas debido a su bajo costo y alta resistencia a la corrosión. También puede ser utilizada como material de partida para la síntesis de magnetita y maghemita, las cuales tienen un mayor interés científico.

La magnetita y maghemita presentan una estructura diferente a la hematita, ya que son miembros del grupo espinela cúbica (óxidos de fórmula ideal $\mathrm{AB}_{2} \mathrm{O}_{4}$, donde $\mathrm{A}$ es un catión divalente y $\mathrm{B}$ un catión trivalente). $\mathrm{El}$ óxido $\mathrm{MgAl}_{2} \mathrm{O}_{4}$, llamado Espinela, da nombre a este tipo de estructuras, que se pueden describir como un empaquetamiento cúbico compacto donde la celdilla elemental cristalográfica (que contienen $8 \quad \mathrm{AB}_{2} \mathrm{O}_{4}$ ) está constituida de la siguiente forma: los 8 átomos de $\mathrm{A}$ están rodeados tetraédricamente por 4 átomos de oxígeno y los 16 átomos de $\mathrm{B}$ están rodeados octaédricamente por 6 átomos de oxígeno. En algunos casos, los átomos A y B están dispuestos de forma diferente, donde la mitad de los átomos de B están ocupando el lugar de A, cuando es así, se formulan como $\mathrm{B}(\mathrm{AB}) \mathrm{O}_{4}$ para diferenciarlos del primer grupo y se denominan espinelas "inversas" [33]). La magnetita es una espinela inversa (Figura 4.b), su fórmula estándar se puede representar como $\mathrm{AB}_{2} \mathrm{O}_{4}$, donde el átomo $\mathrm{A}$ es $\mathrm{Fe}^{2+} \mathrm{y}$ el átomo $\mathrm{B}$ es $\mathrm{Fe}^{3+}$. La maghemita (Figura 4.c) tiene la misma estructura, y puede considerarse como una magnetita deficiente en Fe (II) y se forma por la oxidación topotáctica (sin cambio estructural) de la magnetita [34]. 


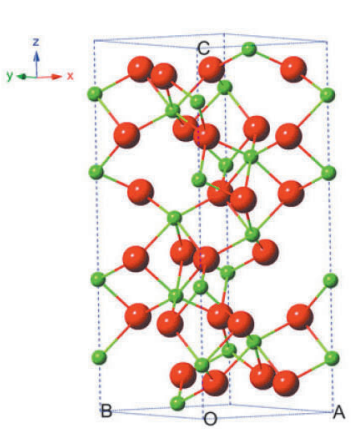

a) Hematita

Romboédrica R3c

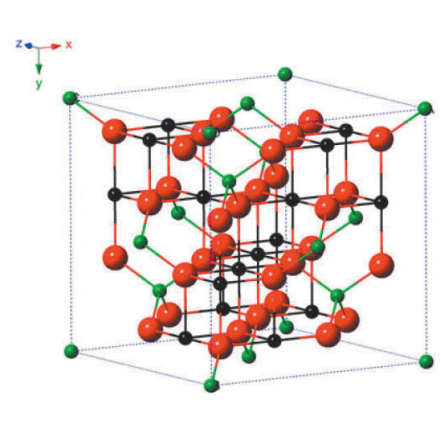

b) Magnetita

Cúbica, Fd3m

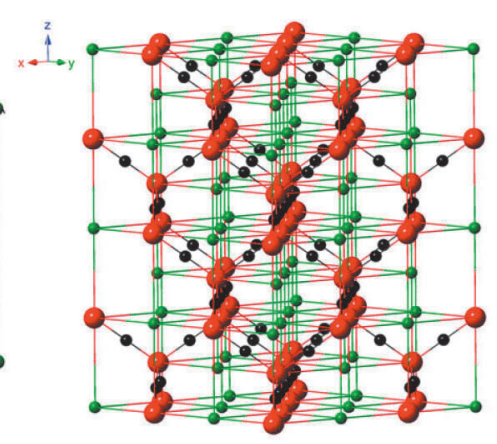

c) Maghemita

Cúbica, $\mathrm{P} 4_{3} 32 /$ Tetragonal, $\mathrm{P} 42_{1}$

Figura 4. Estructura cristalina de los óxidos de hierro. a) hematita, b) magnetita y c) maghemita. (Imagen tomada de Wei Wi y col [35])

Las propiedades magnéticas de los tres óxidos de hierro son bastante similares, aunque la magnetita presenta valores algo más elevados de magnetización. A simple vista, se pueden diferenciar fácilmente por el color, ya que la magnetita es de color negro, la maghemita es marrón y la hematita es roja. A pesar de que estas partículas magnéticas de óxidos de hierro exhiben una menor magnetización de saturación, se prefieren frente a otras nanopartículas metálicas, como el níquel, el cobalto y el hierro, debido al bajo costo, relativa facilidad de síntesis y a la menor toxicidad que presentan las primeras.

Debido a la gran similitud que presentan la maghemita y la magnetita, al sintetizar magnetita suele estar contaminada con maghemita por la tendencia del $\mathrm{Fe}^{2+}$ a oxidarse. Esto se puede evitar realizando la síntesis bajo burbujeo con nitrógeno o algún gas inerte, o realizándola en agua hervida durante una hora previo a la síntesis para eliminar el oxígeno disuelto y manteniendo la condición de ebullición durante la reacción [36] .

\section{SÍNTESIS DE LAS NPMS DE ÓXIDOS DE HIERRO}

Un desafío en el campo de la nanociencia y la nanotecnología es lograr rutas de síntesis eficientes para alcanzar un tamaño controlado, altamente estable y estrecha distribución de fases y composición. Esto ha hecho que sean numerosos los métodos de síntesis descritos, los cuales se pueden clasificar en tres grupo [37, 38]:
- Sintesis en fase líquida: es el grupo de síntesis más utilizado y por tanto las técnicas más comunes serán detalladas en mayor medida en la presente revisión. Estos procesos se basan en la precipitación del material de interés en fase acuosa, donde una etapa de nucleación es seguida por una etapa de crecimiento, $\mathrm{y}$ proporciona un control bastante bueno sobre el tamaño de las partículas. Dentro de este grupo se destacan técnicas como co-precipitación, microemulsión, síntesis hidrotermal y solvotermal, descomposición térmica, sonoquímica, reducción química, métodos con micoondas, entre otros, con los que siguiendo algunos principios, se logra una monodispersión con una desviación estándar menor al $5 \%$.

- Síntesis en fase gaseosa: dentro de este grupo se destacan técnicas como deposición química en fase vapor, descarga de arco y pirolisis laser.

- Sintesis en fase sólida: este método de síntesis es utilizado para obtener NPM recubiertas con carbono, sin embargo, el tamaño y por tanto las propiedades magnéticas de las partículas finales difícilmente pueden controlarse, y partículas superparamagnéticas no se pueden obtener ya que el tamaño de partícula inicial es generalmente mucho mayor del necesario. Dentro de este grupo se destaca la técnica de síntesis por combustión. 


\subsection{Método de co-precipitación}

El método de co-precitación es uno de los más utilizados para la síntesis de NPMs, donde típicamente la magnetita se sintetiza a partir de una solución de sales de hierro $\mathrm{Fe}^{2+}$ y $\mathrm{Fe}^{3+}$ en medio básico (generalmente se agrega hidróxido de sodio o de amonio) e inerte [39]. Para la maghemita el procedimiento es el mismo, pero en presencia de oxígeno. La base desprotona los ligandos hidratados que rodean los cationes de hierro y forma ligandos hidroxilo, para posteriormente producirse la condensación y formación del óxido. La reacción de formación puede escribirse según la ecuación 1 para la magnetita y en la reacción 2 para la maghemita:

$$
\begin{aligned}
& \mathrm{Fe}^{2+}+2 \mathrm{Fe}^{3+}+8 \mathrm{OH}^{-} \rightarrow \mathrm{Fe}_{3} \mathrm{O}_{4}+4 \mathrm{H}_{2} \mathrm{O} \\
& \mathrm{Fe}^{2+}+\mathrm{Fe}^{3+}+\mathrm{O}_{2}+2 \mathrm{OH}^{-} \rightarrow \mathrm{Fe}_{2} \mathrm{O}_{3}+\mathrm{H}_{2} \mathrm{O}
\end{aligned}
$$

Este método es tan común debido a que es un procedimiento en el que la temperatura y el tiempo de reacción son menores comparados con otros métodos como el hidrotermal o descomposición térmica. Además, utiliza un solvente respetuoso del medio ambiente (agua) y se consigue un rendimiento de reacción alto y escalable, lo que hace que sea una manera fácil y conveniente de sintetizan NPMs [40].

Debido a que de los parámetros de síntesis de las NPMs, dependen las propiedades físicas y químicas como el tamaño, cristalización y composición final de las mismas, muchos estudios se han realizado para identificar cuáles son las condiciones que más influyen y de qué modo lo hacen, donde se destacan la naturaleza de las sales utilizadas (por ejemplo, cloruros, sulfatos, o nitratos), relación molar $\mathrm{Fe}^{2+} / \mathrm{Fe}^{3+}$, condiciones de reacción como la temperatura y el $\mathrm{pH}$, tipo y cantidad de base, velocidad de mezcla, fuerza iónica de los medios, secuencia de adición y burbujeo de gas inerte [39]. A continuación analizaremos algunos de estos parámetros según lo reportado.

Un estudio realizado por Lucia Babes y col [41] en el que analizan la influencia de diferentes parámetros en el tamaño y composición de nanopartículas de óxido de hierro determinó que uno de los factores más importantes es la relación molar entre $\mathrm{Fe}^{2+} / \mathrm{Fe}^{3+}$, ya que la formación del núcleo se produce sólo en una relación molar cercana a 0,5 , así, en presencia de una sola clase iónica o de una relación inferior a 0,4 se da la formación de coloidales, por ejemplo, si se usa solo $\mathrm{Fe}^{3+}$, conduce a preparaciones parecidas al hierro paramagnético y para valores de esta relación superiores a 0,6 , el tamaño medio de las partículas aumenta significativamente.

De igual forma, reportaron que otros factores bastante determinantes en el tamaño de partícula son el $\mathrm{pH}$ y la fuerza iónica del medio de precipitación, ya que el tamaño disminuye de forma significativa con el aumento del $\mathrm{pH}$, siendo ideal trabajar a $\mathrm{pH}$ entre 11 y 13 y una fuerza iónica en un área entre 1 y $3 \mathrm{M}$ provocando una casi estabilidad de los tamaños, explicada por la alta carga inducida que genera una caída superficial de la energía.

El pH también es un factor determinante en la especie magnética sintetizada y el mecanismo de reacción, esto lo concluyeron Faiyas y col [42]. Ellos analizaron muestras sintetizadas a diferentes $\mathrm{pH}$ por espectroscopía de fotoelectrones de rayos $\mathrm{X}$ (XPS), encontrando que si se tienen valores de $\mathrm{pH}$ entre 6 y 9, la especie predominante es maghemita y a un valor de $\mathrm{pH} 11$ y libre de oxígeno, se produce solo magnetita. La magnetita no es muy estable bajo condiciones ambientales y se oxida fácilmente, igualmente ocurre en un medio ácido, donde pasa a ser maghemita, aunque dado que la maghemita también tiene propiedades magnéticas, dependiendo de la aplicación para la que se requiera, este puede ser un problema menor. De igual forma, el $\mathrm{pH}$ adecuado para la rápida formación de $\mathrm{Fe}_{3} \mathrm{O}_{4}$ se logra mediante la adición en exceso de la base. Shen y col [43] observaron que el producto presentaba un color parduzco, indicación de la presencia de $\mathrm{Fe}_{2} \mathrm{O}_{3}$, sí el $\mathrm{NH}_{4} \mathrm{OH}$ añadido era insuficiente.

Así mismo, el $\mathrm{NH}_{4} \mathrm{OH}$ favorece una mejor cristalinidad y un tamaño más pequeño, con la consecuente mayor magnetización de saturación en comparación a cuando se utiliza $\mathrm{NaOH}$ como base. Otros estudios han demostrado que los valores de magnetización de saturación de las muestras sintetizadas con sales ferrosas y férricas de sulfato y cloruro fueron de 46,7 y $55,4 \mathrm{emu} \mathrm{g}^{-1}$ respectivamente, demostrando que la naturaleza 
de las sales influencia las propiedades del material final. Por otro lado, en la síntesis de $\mathrm{Fe}_{3} \mathrm{O}_{4}$, la precipitación a temperaturas por debajo de $60{ }^{\circ} \mathrm{C}$ produce típicamente un oxihidróxido hidratado amorfo que puede ser fácilmente convertido en $\mathrm{Fe}_{2} \mathrm{O}_{3}$, mientras que a temperaturas de reacción más altas $\left(>80^{\circ} \mathrm{C}\right)$ se favorece la formación de $\mathrm{Fe}_{3} \mathrm{O}_{4}$, como ya se mencionó antes, esto se debe a la eliminación de oxígeno disuelto en el agua evitando la oxidación del hierro.

En la actualidad se estudia el uso de ultrasonido como factor controlador del tamaño de las partículas. Thanh Quang Bui y col [44] emplearon ultasonido durante todo el proceso de síntesis de las NMPs de magnetita y determinaron que los materiales exhibieron nanocristalitas de dominio único con una morfología esférica uniforme, una distribución de tamaños estrecha (aprox. $10 \mathrm{~nm}$ ) y despreciable campo coercitivo ( $\sim 5$ Oe). Esto se debe, según sus conclusiones, a que a las frecuencias típicas de las irradiaciones por ultrasonidos de $20 \mathrm{KHz}$ a $15 \mathrm{MHz}$, se generan burbujas acústicas que funcionan como puntos para la formación, crecimiento y colapso implosivo de las nanopartículas, creando una condición de reacción especial cerca del límite de la interfaz liquido/solido, presentando el uso del ultrasonido como otro factor a tener en cuenta en la síntesis controlada de NMPs.

\subsection{Método de microemulsión}

El procedimiento de microemulsión ha sido utilizado ampliamente en los últimos 20 años para sintetizar materiales como carboxilatos metálicos y sus óxidos metálicos correspondientes, nanopartículas de aleación metal/metal de alta calidad, nanopartículas semiconductoras y estructuras núcleo-cáscara (núcleo@cáscara), entre otras, con una distribución de tamaños pequeña y controlada [45].

La técnica consiste en mezclar agua, aceite y un surfactante. Cuando la fase oleaginosa está en mayor cantidad y la fase acuosa se dispersa como microesferas rodeadas por las moléculas del surfactante, el método se conoce como micelas inversas o como agua en aceite (w/o). Cuando es la fase acuosa la que está en mayor medida, se conoce como micelas normales o aceite en agua $(\mathrm{o} / \mathrm{w})$. En ambos casos la concentración del surfactante debe ser superior a la concentración crítica de las micelas y el tamaño de la micela va a depender de la relación molar surfactante/agua o surfactante/aceite, según sea el tipo de método utilizado. Las nanopartículas son producidas por la microemulsión, que desempeña un papel de molde o nano creador y su tamaño va a depender de la presencia de agentes estabilizantes adicionales y la concentración de los reactivos $[46,47]$.

El pH también cumple una función importante en este método de síntesis, donde tiene una implicación directa sobre la forma de las partículas, a esta conclusión llegaron Z. H. Zhou y col [48] quienes informaron el uso de una emulsión de aceite en agua para sintetizar NPMs de $\mathrm{Fe}_{3} \mathrm{O}_{4}$ con ciclohexano como fase oleosa, polioxietileno (5) nonilfenil éter (NP5) y Polioxietileno (9) nonilfenil éter (NP9) como surfactantes y soluciones de las sales $\mathrm{FeSO}_{4} /$ $\mathrm{Fe}\left(\mathrm{NO}_{3}\right)_{3}$ como precursores en fase acuosa. Encontraron que a $\mathrm{pH}=8,5$ se forman partículas con forma de varillas afiladas, a $\mathrm{pH}=10,5 \mathrm{se}$ forma una mezcla de las partículas con formas esféricas y varillas afiladas y a $\mathrm{pH}=13,5$ se forman solo partículas esféricas. Esto podría estar relacionado con la forma que adopta la micela según el valor de $\mathrm{pH}$ del medio. El rendimiento de la síntesis fue de $88 \%$ y un tamaño de partícula promedio de $<10 \mathrm{~nm}$. Finalmente concluyeron que una fase fuertemente alcalina favorece la formación de $\mathrm{Fe}_{3} \mathrm{O}_{4}$, mientras que una alcalinidad baja favorece la formación de $\alpha-\mathrm{FeOOH}$.

Por otra parte, la elección del surfactante es importante, ya que podría influenciar en la estabilidad y variación del tamaño de micelas, y por lo tanto, en el rango de tamaño de las NPMs de óxido de hierro formadas. Bumajdad y col [49] investigaron el uso de un sistema de surfactantes mixto de di-n-didodecildimetilamonio bromuro (DDAB) con una pequeña cantidad de Brij35 no iónico, y encontraron que se podía lograr una alta proporción de agua a surfactante de 50 , en comparación con las micelas inversas. Ellos razonaron que la carga positiva del grupo de la cabeza del surfactante catiónico aseguraba la solubilidad en masa del catión del metal inorgánico, mientras que la larga cadena del óxido de polietileno del surfactante no iónico facilitaba una mayor estabilidad estérica, dando como resultado un sistema de micelas de alta estabilidad y baja sensibilidad térmica. El DDAB también 
mejoró la estabilidad de la microemulsión, y se prepararon NPMs de $\gamma-\mathrm{Fe}_{2} \mathrm{O}_{3}$ tan pequeñas como $3 \mathrm{~nm}$.

\subsection{Síntesis hidrotermal}

La síntesis hidrotérmica se refiere a la obtención por reacciones químicas de monocristales de minerales en una solución caliente y altas presiones en autoclave. Básicamente, el mecanismo de las reacciones hidrotérmicas sigue un modelo de nucleación líquida, donde la alta temperatura es beneficiosa para mejorar la velocidad de nucleación y acelerar el crecimiento de las nuevas partículas, lo que da resultado a su pequeño tamaño [50]. La hidrólisis y oxidación son unas de las principales vías para obtener nanopartículas en condiciones hidrotérmicas, y otra vía es la neutralización de hidróxidos de metales híbridos. Generalmente se usa un precursor ferroso como material de partida contrario a la mezcla estequiométrica utilizada en el método de co-precipitación y en general, las partículas tienen un tamaño relativamente mayor en comparación con la co-precipitación. Este método es relativamente poco explorado para la síntesis de nanopartículas magnéticas, aunque permite la síntesis de nanopartículas de alta calidad $[38,46]$.

Aun así, mediante el control de los parámetros, incluida la temperatura, el tiempo de reacción, la concentración de los reactivos y las características del disolvente, se puede optimizar el parámetro geométrico de las nanopartículas. En un estudio realizado por Ahlam Nemati y col [51] sintetizaron y caracterizaron nanopartículas de magnetita en presencia de $2,2^{\prime}, 2^{\prime \prime}, 2^{\prime \prime}$ - (etano1,2-diilbis(azanetriil)) tetra acetohidracida como agente reductor en la reacción hidrotermal, encontrando que las nanopartículas de $\mathrm{Fe}_{3} \mathrm{O}_{4}$ tenían un rango de diámetros entre $15-21 \mathrm{~nm}$ y que las diferentes condiciones de reacción, como la concentración de ligando, la temperatura y el tiempo de reacción tuvieron un efecto leve sobre el tamaño de las nanopartículas sintetizadas.

Recientemente, se han reportado estudios donde fabrican películas de $\mathrm{Fe}_{3} \mathrm{O}_{4}$ mediante síntesis hidrotermal a baja temperatura. Dichas películas son utilizadas en la absorción selectiva de luz solar. En el estudio determinaron que la morfología de la superficie y el espesor de las películas pueden ajustarse por los factores de concentración alcalina y tiempo de reacción [52]. También por esta técnica se han sintetizado nanocompuestos de $\mathrm{Fe}_{3} \mathrm{O}_{4}$ soportadas en aerogel de celulosa, dicho material presentó una capacidad de respuesta magnética alta que permite una separación fácil del medio de reacción mediante la aplicación de un campo magnético externo [53].

\subsection{Síntesis solvotermal}

El principio de la síntesis solvotérmica es similar a la síntesis hidrotérmica, pero se utiliza un disolvente orgánico como medio de dispersión en lugar de agua. El uso de solventes orgánicos no solo expandió el alcance de la selección de materiales, sino que también permitió realizar reacciones que no son adecuadas para el método hidrotermal. Al igual que las técnicas anteriormente mencionadas, la elección del solvente, precursor, temperatura y tiempo de reacción y período de envejecimiento son cruciales en el tamaño de partícula, morfología y distribución. Como ventaja adicional, la síntesis solvotérmica puede evitar efectivamente la adsorción de aniones y cationes en la superficie de las nanopartículas, evitando así la contaminación típica en otros métodos como lo son hidrotermal y co-precipitación [54].

Una síntesis común de ferritas microesféricas cristalinas por esta técnica, consiste en la mezcla de cloruro férrico $\left(\mathrm{FeCl}_{3}\right)$, etilenglicol (agente reductor), acetato de sodio (inhibidor de aglomeración) y polietilenglicol (agente tensioactivo), obteniendo una solución transparente que se calienta dentro de un autoclave a $200{ }^{\circ} \mathrm{C}$ durante $8-72 \mathrm{~h} \mathrm{[55].} \mathrm{Como} \mathrm{desventaja,}$ este método tiene una velocidad de reacción muy baja, por lo que generalmente se acompaña de otra técnica como microondas para aumentar la velocidad de la formación de cristales, ya que la radiación rápida de las microondas induce una ráfaga de "puntos calientes" en la solución precursora, acelerando así la formación de nanocristales de $\mathrm{Fe}_{3} \mathrm{O}_{4}$. En un estudio reportado por Cuiyan $\mathrm{Li}$ y col [56], las nanopartículas de $\mathrm{Fe}_{3} \mathrm{O}_{4}$ preparadas por el método microondassolvotérmico mostraron una mayor magnetización de saturación que la muestra sintetizada por el método solvotérmico convencional, que se puede atribuir al hecho de que la primera tiene tamaños 
de partículas más pequeños que la segunda, además esta técnica combinada permite incluso, disminuir la temperatura de síntesis y el tiempo de reacción.

\section{MODIFICACIÓN DE LASUPERFICIE DE LAS NMPS DE ÓXIDOS DE HIERRO}

Dado que la superficie de las NPMs de óxidos de hierro está cubierta con grupos hidróxido, normalmente son dispersables solo en suspensiones polares. Además, son muy sensibles a la oxidación si se dejan expuestas al aire del ambiente, cambiando drásticamente sus propiedades, esto debido a su tamaño ultra fino, su gran área de superficie específica y su alta reactividad química. Así mismo, no son estables en las duras condiciones necesarias para las reacciones catalíticas que en muchos casos ocurren en medio ácido [57]. Por lo tanto, la modificación de la superficie de las NPMs es un paso esencial para su uso como soportes para catalizadores. Esto ha hecho que existan muchos estudios sobre materiales y metodologías para modificar su superficie de tal forma que se les confiera estabilidad y les permita dispersarse con facilidad en diferentes medios, incluso a altas temperaturas y en la que se pueda inmovilizar la fase activa del catalizador, pero que además conserve sus propiedades magnéticas y se pueda aplicar a escala industrial. Los materiales más usados y que cumplen con estas condiciones a menudo son el óxido de silicio, ligandos de coordinación y polímeros orgánicos o inorgánicos que encapsulan las NPMs [58].

\subsection{Materiales inorgánicos.}

\subsubsection{Encapsulación de NPMs de óxidos de hierro con óxido de silicio}

Dentro de los materiales utilizados para encapsular NPMs, el $\mathrm{SiO}_{2}$ ha atraído una atención especial en primer lugar, porque las capas de sílice sobre la superficie de las NPMs disminuyen la atracción dipolar magnética, lo que favorece su dispersión en medios líquidos y los protege de la lixiviación en un ambiente ácido. En segundo lugar, debido a la existencia de abundantes grupos silanoles, la capa de sílice, sirve de anclaje de diferentes materiales y grupos funcionales y así, inmovilizar, por ejemplo, catalizadores homogéneos. Finalmente, la capa de sílice proporciona una superficie químicamente inerte para ser utilizada en sistemas biológicos. Estas características se pueden modificar a través de la variación del espesor de la capa $[12,57,59]$.

Se han explorado en mayor medida dos técnicas diferentes para recubrir con sílice las superficies de partículas de óxido de hierro. El primero es el método Stöber, reportado por Werner Stöber y Arthur Fink en 1968 [60], siendo aún el método de síntesis de sílice más utilizado porque permite un tamaño monodisperso de partículas, su bajo costo, condiciones suaves de reacción y libre de surfactantes, entre otras ventajas. Este método es un ejemplo de un proceso sol-gel en el cual la sílice se forma in situ a través de la hidrólisis y condensación de un precursor (típicamente TEOS) en una solución alcohólica (generalmente metanol o etanol) y amoniaco como catalizador (Figura 5).

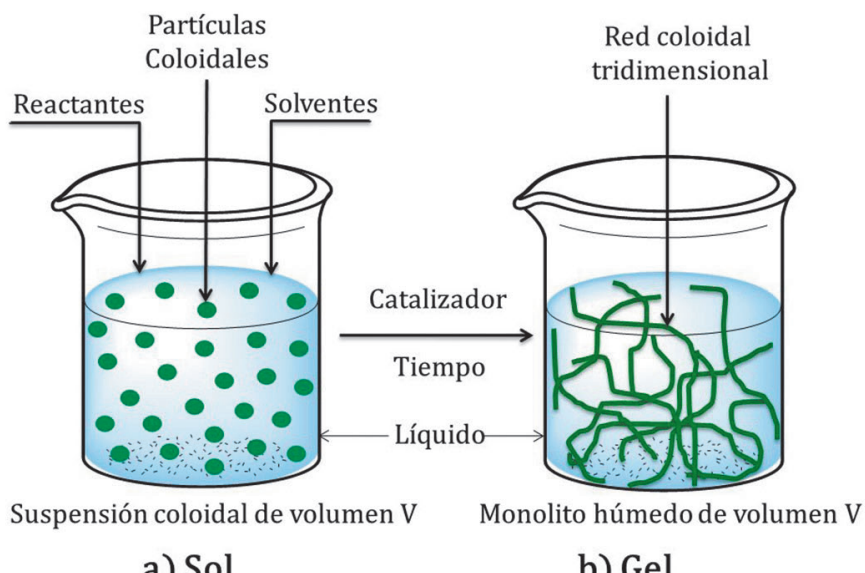

a) Sol

Figura 5. Representación gráfica del método sol-gel. 
Una representación de esta reacción se muestra en la Figura 6. Las moléculas resultantes se unen para formar estructuras más grandes, donde el tamaño se puede controlar dependiendo de las condiciones de reacción (relación molar de reactivos, cantidad de base, velocidad de mezcla, etc) $[61]$.

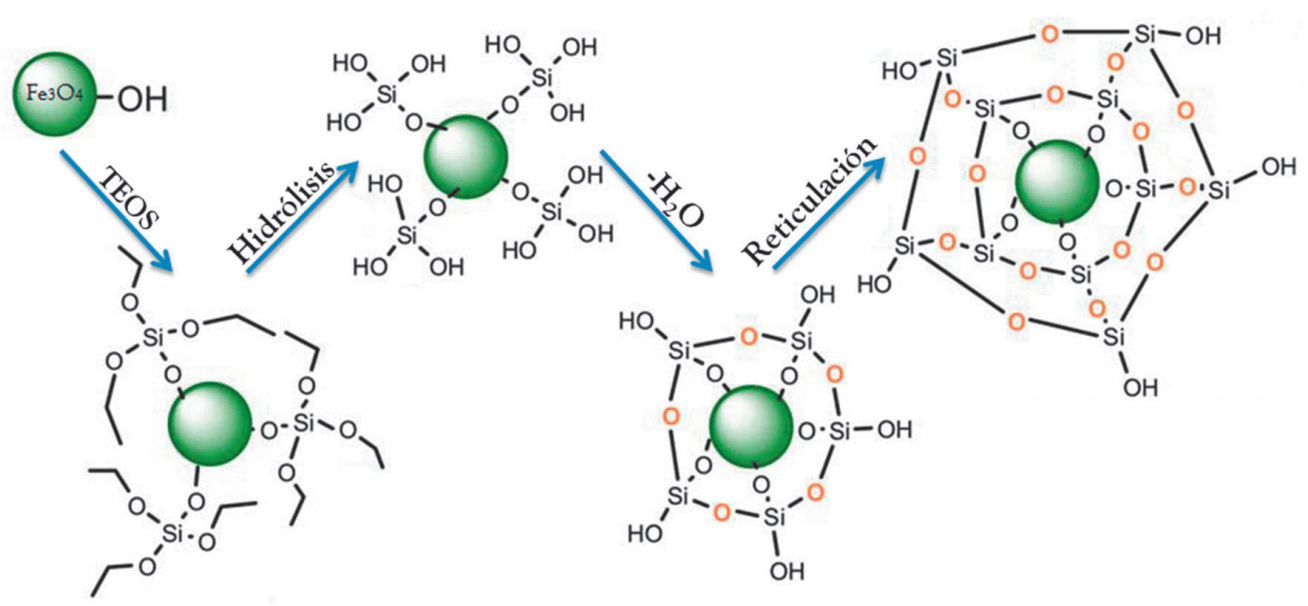

Figura 6. Representación simplificada de la hidrólisis y condensación de TEOS sobre las NPMs en el proceso Stöber. (Imagen modificada de G.H. Du et al 2006 [18]).

La reacción consiste en la obtención de soles mediante la hidrólisis de los precursores catalizada por una base para formar especies $=\mathrm{SiOH}$ sumamente reactivas. Estas especies continúan reaccionando mediante la alcoxolación (reacción donde se forman puentes oxo $\mathrm{Si}-\mathrm{O}-\mathrm{Si}$ a través de la eliminación de una molécula de alcohol), o la oxolación (reacción análoga a la alcoxolación, excepto por que la molécula saliente en lugar de alcohol es agua), estas reacciones también se conocen como condensación. La hidrólisis de los grupos etóxidos y la posterior condensación conduce a la reticulación, el cual es un proceso de una etapa ya que estas dos reacciones ocurren juntas en un único recipiente de reacción [62]

El segundo método es la microemulsión [63], del que ya se habló anteriormente. Para el caso de los recubrimientos de las NPMs con el óxido de silicio, comúnmente, las NPMs son previamente recubiertas por un ligando hidrofóbico, dispersados en un medio orgánico. Posteriormente se agrega una solución acuosa alcalina, que contiene el surfactante y la mezcla es tratada generalmente con ultrasonido formando así la microemulsión. Finalmente un precursor de sílice es agregado, generalmente TEOS y se deja reaccionar durante un determinado tiempo. Así, microsferas de sílice mesoporosa con núcleo magnético son obtenidas, donde el crecimiento, grosor y regularidad de la capa de sílice depende de los parámetros experimentales como la concentración de TEOS, cantidad de agua, tiempo de reacción, tipo de surfactante, entre otros. Como desventaja, este método requiere de un mayor esfuerzo para separar las esferas de sílice, de la gran cantidad de tensoactivos asociados con el sistema de microemulsión.

Por otro lado, la fase activa se puede soportar de dos formas: 1) directamente sobre el óxido de silicio, como es el caso de los estudio realizado por Ding Hong y col [64] que sintetizaron NPMs de $\mathrm{Fe}_{3} \mathrm{O}_{4} @ \mathrm{SiO}_{2}$ funcionalizadas con óxido de cerio mesoporoso $\left(\mathrm{Fe}_{3} \mathrm{O}_{4} @ \mathrm{SiO}_{2} @ \mathrm{mCeO}_{2}\right)$ y Li Lai y col [65] que inmovilizaron óxido de lantano sobre $\mathrm{Fe}_{3} \mathrm{O}_{4} @ \mathrm{SiO}_{2}$. Los dos materiales fueron evaluados en la adsorción eficiente de fosfato en muestras acuosas. 2) El óxido de silicio puede tener modificaciones sobre su superficie como agregados de otras partículas tales como aminas, y ligandos organometálicos [66-68] a los cuales se une la fase activa. En un estudio reportado por L. Tzounis y col [69], sintetizaron un material denominado $\mathrm{Fe}_{3} \mathrm{O}_{4} @ \mathrm{SiO}_{2} @ \mathrm{Ag}, \quad$ donde las partículas de $\mathrm{Fe}_{3} \mathrm{O}_{4} @ \mathrm{SiO}_{2}$ se modificaron con polietilenimina (PEI) para introducir grupos amina, los cuales fueron coordinados con los iones $\mathrm{Ag}^{+}$suministrados por una solución acuosa de nitrato de plata. Un esquema de la obtención del catalizado se muestra en la Figura 7. 


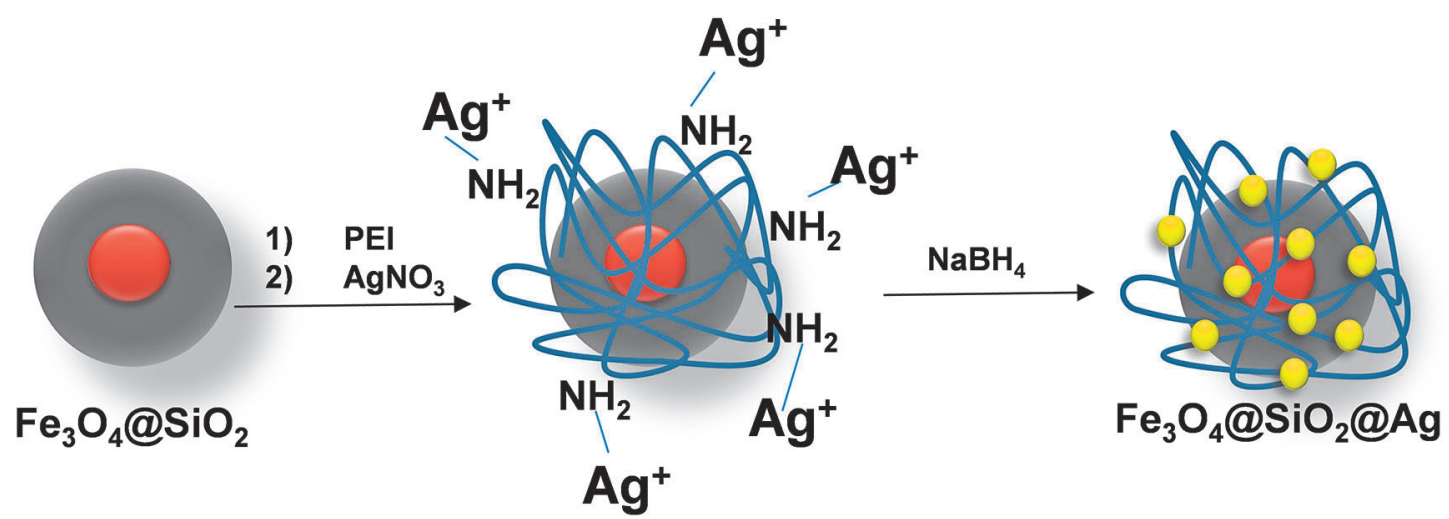

Figura 7. Ilustración esquemática para la funcionalización de partículas de $\mathrm{Fe}_{3} \mathrm{O}_{4} @ \mathrm{SiO}_{2}$ con nanopartículas de $\mathrm{Ag}$ utilizando PEI como ligando de iones de plata.

Como ejemplos de ligandos organometálicos, M. Esmaeilpour y col [70] funcionalizaron la superficie de la sílice con complejos de bases de Shiffcondiferentesionesmetálicosmediantevarios pasos. En primer lugar, sintetizaron nanoesferas de $\mathrm{Fe}_{3} \mathrm{O}_{4} @ \mathrm{SiO}_{2}$ con TEOS como fuente de sílice y PVA como surfactante. Luego, sintetizaron los complejos de bases de Schiff a partir de la reacción entre la base y los diferentes acetatos metálicos $\left[\mathrm{Co}(\mathrm{OAc})_{2}, \quad \mathrm{Mn}(\mathrm{OAc})_{2}, \quad \mathrm{Ni}(\mathrm{OAc})_{2}\right.$, $\mathrm{Cu}(\mathrm{OAc})_{2}, \mathrm{Hg}(\mathrm{OAc})_{2}, \mathrm{Cr}(\mathrm{OAc})_{3}$ y $\left.\mathrm{Cd}(\mathrm{OAc})_{2}\right]$ y finalmente se inmovilizaron sobre la superficie de $\mathrm{Fe}_{3} \mathrm{O}_{4} @ \mathrm{SiO}_{2}$ (Figura 8). La capacidad catalítica de los materiales fue evaluada en la conversión de aldehídos en sus correspondientes compuestos 1,1-diacetatos, encontrando que son eficientes, incluso en condiciones suaves y sin disolventes a temperatura ambiente, según el siguiente orden se rendimiento: $\mathrm{Cr}$ (III) $>\mathrm{Ni}$ (II) $>\mathrm{Zn}$ (II) $>\mathrm{Cu}$ (II) $>\mathrm{Mn}$ (II) $>\mathrm{Co}$ (II) $>\mathrm{Cd}$ (II) $>\mathrm{Hg}$ (II).

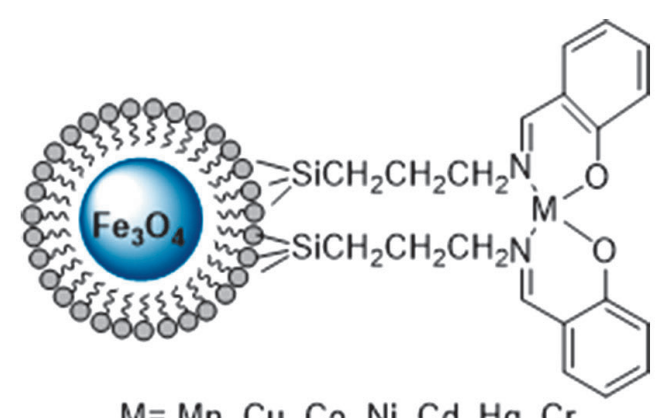

$\mathrm{M}=\mathrm{Mn}, \mathrm{Cu}, \mathrm{Co}, \mathrm{Ni}, \mathrm{Cd}, \mathrm{Hg}, \mathrm{Cr}$

Figura 8. Esquema del catalizador $\mathrm{Fe}_{3} \mathrm{O}_{4} @ \mathrm{SiO}_{2}$ funcionalizado con el complejo de base de Schiff de diferentes iones metálicos sobre.

Éste grupo de investigación también sintetizó catalizadores magnéticos funcionalizados con bases de Schiff, en esta ocasión con paladio como centro activo y los estudiaron en reacciones de acoplamiento de Sonogashira-Hagihara, la cual es una de las reacciones de formación de enlaces carbono/carbono más utilizadas en Química Orgánica. En este caso, estudiaron la reacción de acoplamiento directo de haluros de arilo con fenilacetileno, encontrando que el catalizador muestra ventajas notables como la simplicidad de operación, excelentes rendimientos, cortos tiempos de reacción, fácil separación magnética y reciclabilidad [71].

Los líquidos iónicos son otro tipo de fase activa que ha sido soportada sobre $\mathrm{Fe}_{3} \mathrm{O}_{4} @ \mathrm{SiO}_{2}$. Hui Wan y col [72] desarrollaron catalizadores verdes y reutilizables para la preparación de biodiesel, conformados por 3-sulfopropil-1-(3-propil trimetoxisilano) hidrógeno de sulfato imidazolio ([SO $\left.\mathrm{SO}_{3} \mathrm{H}-\mathrm{PIM}-\mathrm{TMSP}\right] \mathrm{HSO}_{4}$ ) sobre nanopartículas de $\mathrm{Fe}_{3} \mathrm{O}_{4}$ modificadas con sílice mesoporosa. El catalizador presentó una conversión de $93,5 \%$ en la esterificación del ácido oleico, fácil separación y varios ciclos de reutilización.

\subsubsection{Funcionalización NPMs de óxidos de hierro con metales y óxidos metálicos}

Los catalizadores de metales y óxidos metálicos inmovilizados sobre NPMs se dividen en catalizadores de complejos metálicos y catalizadores de nanopartículas metálicas. Estos catalizadores se preparan generalmente a través de dos procedimientos: (1) reacción directa de complejos metálicos con NPMs; (2) coordinación de precursores de complejos metálicos con NPMs modificados con ligandos quelantes [58]. 
Uno de los óxidos metálicos más usados es el óxido de magnesio $\mathrm{MnO}_{2}$ ya que tiene un área de superficie alta, una fuerte capacidad de oxidación/ adsorción y una buena estabilidad en condiciones ácidas. Así, al recubrir $\mathrm{NPMs}_{\mathrm{de}} \mathrm{Fe}_{3} \mathrm{O}_{4}$ con $\mathrm{MnO}_{2}$, el material resultante presenta aplicaciones como adsorbentes de iones metálicos y colorantes en tratamientos de aguas residuales [73, 74], con la facilidad de separación que otorga la magnetita. Qingxiang Yang y col [75] sintetizaron compuestos magnéticos recubiertos con $\mathrm{MnO}_{2}$ $\left(\mathrm{Fe}_{3} \mathrm{O}_{4} @ \mathrm{MnO}_{2}\right)$ mediante un proceso hidrotermal simple. El tamaño de partícula de los compuestos obtenidos está en el rango de 300 a $400 \mathrm{~nm}$ y presentó actividad en la eliminación selectiva del colorante rojo Congo de una solución acuosa que contenía varios tintes (rojo Congo (CR), azul de metileno (MB), metil naranja (MO), violeta cristal (CV) y rodamina $\mathrm{B}(\mathrm{RB})$ ), esto, debido a las interacciones entre la superficie cargada positiva del compuesto $\mathrm{Fe}_{3} \mathrm{O}_{4} @ \mathrm{MnO}_{2}$ y las moléculas del colorante aniónico.

Otro ejemplo de óxidos utilizados es el óxido de niobio $\left(\mathrm{Nb}_{2} \mathrm{O}_{5}\right)$, también conocido como niobia, que al recubrir las NPMs, cumple una doble función, primero, protege las nanopartículas y segundo, genera la función ácida necesaria para depositar la fase activa del catalizador. Dentro de las ventajas de este óxido se destaca su acidez, actividad, estabilidad e insolubilidad, requeridas para catalizar reacciones en medios acuosos. Además, contiene fuertes sitios ácidos de Lewis y Brönsted, y como soporte, el óxido de niobio puede provocar fuertes interacciones con metales que cumplen la función de centros activos para diferentes reacciones [76]. Así, Cristina Opris y col [77] sintetizaron catalizadores de magnetita recubierta con óxido de niobio y cobalto como fase activa $\left(\mathrm{Fe}_{3} \mathrm{O}_{4} @ \mathrm{Nb}_{2} \mathrm{O}_{5} @\right.$ $\mathrm{Co}$ ) para catalizar la división del enlace $\mathrm{C}-\mathrm{C}$ en la fragmentación de lignina. Como resultado, se logró una fragmentación completa de la lignina donde los fragmentos dominantes eran aquellos que contenían moléculas C20-C28. Este comportamiento catalítico se explica sobre la capacidad del óxido de niobio para catalizar la hidrólisis ácida de los extremos $\beta$-O-4' y del Co para romper los enlaces C-C a través de la hidrogenólisis. La optimización de la composición del catalizador indicó una carga del $4 \%$ en peso de Co como óptima y una temperatura de reacción de $180{ }^{\circ} \mathrm{C}$.
También se han realizado estudios de magnetita funcionalizada con metales como paladio, níquel, cobalto, aluminio y cesio y se han evaluado en diferentes reacciones orgánicas. Así, Jun Liu y col [78] sintetizaron un catalizador de $\mathrm{Pd} / \mathrm{Fe}_{3} \mathrm{O}_{4}$ por el método de coprecipitación y lo evaluaron en la reacción de reformado en fase acuosa de etilenglicol (EG) a $\mathrm{H}_{2}$, el cual mostró un rendimiento catalítico superior en términos de actividad, selectividad y estabilidad que los catalizadores de Pd soportado en diferentes óxidos metálicos preparados por impregnación de humedad incipiente. El excelente rendimiento catalítico del catalizador se atribuyó al efecto sinérgico mejorado entre las pequeñas nanopartículas de Pd y la magnetita para promover la reacción de cambio de agua y gas, que es el paso determinante de la velocidad en la reacción sobre catalizadores basados en Pd.

Este tipo de catalizadores también han sido evaluados en la reacción de acoplamiento cruzado de Suzuki y en la reducción de nitroarenos en condiciones suaves (presión de $\mathrm{H}_{2}$ en el balón y temperatura ambiente), encontrando altos rendimientos, recuperación eficaz y que son reutilizables varias veces con actividad catalítica sostenida [79]. Por su parte, Jianpeng Shang y col [80] prepararon una serie de catalizadores de óxido de hierro magnético que contenían diferentes concentraciones de níquel, mediante el método de coprecipitación y se probaron en la síntesis de carbamatos $\mathrm{N}$-sustituidos empleando aminas y carbamatos de alquilo. Bajo las condiciones de reacción optimizadas, varios carbamatos $\mathrm{N}$-sustituidos fueron sintetizados exitosamente con un rendimiento de 90-98\%. Bing Yang y col [81] prepararon una serie de materiales de $\mathrm{Fe}_{3} \mathrm{O}_{4}$ dopados con cobalto, también por el método de coprecipitación química en solución acuosa. El catalizador permitió que la hidrogenación de cloronitrobencenos a cloroanilinas (CA) procediera a bajas temperaturas en agua y a presión atmosférica, dando como resultado aproximadamente un $100 \%$ de rendimiento y selectividad. Finalmente, Mostafa Feyzi y col [82] sintetizaron catalizadores de magnetita, aluminio y cesio $\left(\mathrm{Fe}_{3} \mathrm{O}_{4} / \mathrm{Al} / \mathrm{Cs}\right)$, e investigaron $\mathrm{los}$ efectos de las diferentes relaciones molares $\mathrm{Cs} / \mathrm{Al}$ y $\mathrm{Cs} / \mathrm{Fe}$, y las condiciones de calcinación sobre el rendimiento catalítico de los materiales para la producción de FAME (éster metílico de 
ácidos grasos) de biodiesel. Encontrando que el catalizador con una relación molar $\mathrm{Cs} / \mathrm{Al}=2.5 / 1$ y $\mathrm{Cs} / \mathrm{Fe}=4 / 1$ era el óptimo para la producción de biodiesel.

\subsubsection{Encapsulación de NPMs de óxido de hierro con polímeros inorgánicos}

Los polímeros son materiales importantes con propiedades ajustables que se pueden usar para modificar la superficie de los nanomateriales. Por esto, se han utilizado ampliamente para funcionalizar y estabilizar las NPMs de óxido de hierro a través de diversas técnicas [83]. Se destacan la polimerización por radicales controlada (CRP) que permite preparar materiales poliméricos con pesos moleculares controlados y la polimerización por transferencia reversible de cadena de adición-fragmentación (RAFT) que permite sintetizar una variedad de polímeros funcionales con diferentes arquitecturas de manera controlada (pesos moleculares predecibles y bajas polidispersidades). Ambas técnicas presentan ventajas significativas, como la aplicabilidad a muchos monómeros polimerizables por radicales, baja contaminación de metales en el polímero final y condiciones de polimerización suaves [84]. Otros métodos incluyen polimerización en suspensión, polimerización en dispersión y polimerización en emulsión [85]. De igual forma, se ha utilizado una gran variedad de polímeros con diferentes grupos funcionales como carboxilato, carboxilo, estireno y alcohol vinílico para preparar y recubrir NPMs [86].

Bin Liu y col [87] desarrollaron un método general para la síntesis de microesferas de polímero con núcleo $\mathrm{Fe}_{3} \mathrm{O}_{4}$ bien definido, diferentes grupos funcionales y una fuerte magnetización. En este enfoque, las microesferas de $\mathrm{Fe}_{3} \mathrm{O}_{4}$ preparadas se recubrieron directamente con una capa de polímero a través de la interacción de enlaces de hidrógeno entre las microesferas de magnetita y el oligómero. Además, propiedades como la hidrófilicidad o hidrófobicidad se pudieron modificar variando los grupos funcionales tales como carboxilo, hidroxilo, amida y éster encapsulados sobre la superficie de las microesferas de magnetita. El grosor de la capa del polímero se ajustó según la cantidad de monómero agregado y como fase activase se estudió una serie de nanopartículas metálicas como Ag, Pt y Au. Guangyu Liu y col [88] sintetizaron microesferas huecas que contienen NPMs móviles en su cavidad, lo que resulta en una estructura interesante con propiedades novedosas diferentes de las microesferas con núcleo compacto.

\subsubsection{Encapsulación de NPMs de óxidos de hierro con polímeros orgánicos}

El recubrimiento de NPMs con polímeros orgánicos les confiere varias propiedades deseables tales como excelente estabilidad térmica, cantidad de carga de extensión del catalizador inmovilizado, inercia química, ausencia de lixiviación del catalizador y facilidad para la posterior funcionalización de funcionalización sucesiva.

Un ejemplo de un polímero orgánico es el quitosano (QS), el cual es un amino polisacárido lineal natural, obtenido por desacetilación alcalina de la quitina. La quitina es el segundo polisacárido más abundante después de la celulosa y es el componente principal del exoesqueleto protector de los crustáceos como cangrejos, camarones, langostinos, langostas y paredes celulares de algunos hongos como el aspergillus y el mucor [89]. El quitosano se destaca por un conjunto especial de propiedades que incluyen disponibilidad, seguridad, no toxicidad, biocompatibilidad, biodegradabilidad y baja inmunogenicidad. Se compone de copolímeros de glucosamina y $\mathrm{N}$-acetil-glucosamina, donde hay un grupo amino primario y dos grupos hidroxilo libres para cada unidad de construcción C6 que sirven como sitios de coordinación para una variedad de modificaciones químicas. Como resultado, los catalizadores a base de quitosano han recibido mucha atención para llevar a cabo diferentes tipos de reacciones orgánicas, como oxidación, acoplamiento $\mathrm{C}-\mathrm{C}$, reacciones multicomponente, entre muchas otras [90].

A. Naghipour y col[91] sintetizaron un catalizador ecológico basado en paladio soportado sobre nanopartículas de magnetita recubiertas con quitosano. La actividad catalítica se evaluó en la reacción de acoplamiento de Suzuki-Miyaura, en haluros de arilo y ácido fenilborónico. Además, se encontró que el catalizador mostró una alta actividad para la reacción de Mizoroki-Heck de 
haluros de arilo y acrilato de n-butilo. Se aisló fácilmente de la mezcla de reacción y se usó en cinco ciclos consecutivos sin pérdida significativa en la actividad.

Por otra parte, la celulosa es otro biopolímero utilizado para funcionalizar NPMs. Es un polisacárido insoluble en agua relativamente homogéneo, constituido por varios miles de moléculas de D-glucosa $\left(\mathrm{C}_{6} \mathrm{H}_{10} \mathrm{O}_{5}\right)_{\mathrm{n}}$ unidas por enlaces $\beta$-1,4-glucosídicos no ramificados, que junto con la hemicelulosa, cumplen la función de dar dureza y soporte a las plantas, siendo el componente principal de la pared celular vegetal correspondiente a una fracción cercana al 50\%, además, como ya se mencionó anteriormente, es el biopolímero más abundante sintetizado por la naturaleza [92]. Debido a su biodegradabilidad, ser un recurso abundante, su bajo costo, su hidrofilicidad, su alta resistencia, su capacidad de renovación y sus propiedades mecánicas únicas, es muy utilizada en diferentes estudios de investigación [93]. Saeid Zarei y col [94] extrajeron celulosa de las algas Cystoseria myricaas y sintetizaron compuestos $\mathrm{Fe}_{3} \mathrm{O}_{4}$ Nanocelulosa por los métodos de hidrólisis ácida, co-precipitación y sol-gel y los evaluaron en la captación de $\mathrm{Hg}$ (II) en aguas residuales donde determinaron la cinética de la reacción, la energía de activación por adsorción, la termodinámica del proceso y la energía libre de Gibbs. Determinaron que la celulosa es un absorbente más estable y apropiado que otros polímeros similares como agar, quitina o el oligosacárido manano.

\section{HETEROPOLIÁCIDOS COMO FASE ACTIVA SOPORTADOS SOBRE NPMS DE ÓXIDOS DE HIERRO}

La catálisis por heteropoliácidos (HPAs) y compuestos relacionados de polioxometalatos (POMs, especies heteropolianiónicas) es un campo de gran importancia debido a sus ventajas tales como baja volatilidad, baja corrosividad, alta actividad, flexibilidad y estabilidad térmica, además, los HPAs presentan una acidez Bronsted muy fuerte, acercándose a la región de un superácido, y son eficientes oxidantes, que exhiben rápidas transformaciones redox bajo condiciones bastante suaves que los postulan como catalizadores económicos y ecológicos utilizados en diversas reacciones en fase líquida homogénea y como remplazo de catalizadores convencionales como el ácido sulfúrico y clorhídrico [95-97]. Sin embargo, se ve la necesidad de ampliar dicha área soportándolos, obteniendo catalizadores activos para una mayor cantidad de aplicaciones y facilitando su separación del medio de reacción, permitiendo su reutilización [98].

Con los avances en nanociencia y nanotecnología y el auge de los sistemas de nanocatalizadores magnéticamente reciclables en la catálisis heterogénea, se busca soportar HPAs sobre diferentes sistemas de NPMs. Los HPA más estudiados son los que tienen estructura tipo Keggin. Así, Aiguo Kong y col [99] sintetizaron un nanocatalizador ácido recuperable magnéticamente, basado en ácido tungstosilícico (HSiW) soportado sobre magnetita recubierta con quitosano (HSiW-CS@Fe3O4),y fue evaluado en la reacción de acetalización de benzaldehído con etilenglicol, alcanzando un rendimiento del 99\% para el acetal en condiciones de reacción suaves. Lo aislaron fácilmente del medio de reacción, lo reutilizaron 5 veces sin pérdida significativa de su actividad y se confirmó que no se modificaron drásticamente las características fisicoquímicas del catalizador luego de recuperado. Fatemeh Nikbakht y col [100] inmovilizaron ácido tungstofosfórico (HPW) sobre $\gamma$ - $\mathrm{Fe}_{2} \mathrm{O}_{3}$ encapsulado con sílice como catalizador eficiente para la oxidación directa de aminas secundarias a nitronas con peróxido de hidrógeno como agente oxidante. Jiongliang Yuan y col [101] prepararon catalizadores de HPW soportados sobre $\mathrm{Fe}_{3} \mathrm{O}_{4} @ \mathrm{SiO}_{2}$ mediante una técnica de incorporación del ácido durante la síntesis sol-gel del recubrimiento de sílice en lugar del método de impregnación. El rendimiento catalítico de los catalizadores resultantes es comparable al de los heteropoliácidos puros en la reacción de esterificación de etanol con ácido acético.

Por otro lado, aunque en la mayoría de los materiales sintetizados, la fase activa se inmoviliza directamente sobre el óxido de silicio, se presentan muchos estudios que incorporan una modificación adicional. Liang Zhao y col [102] prepararon catalizadores de HPW sobre $\mathrm{Fe}_{3} \mathrm{O}_{4} @ \mathrm{SiO}_{2}$ funcionalizada con (3-aminopropil) trietoxisilano (APTES) como agente de acoplamiento con el HPA. Durante la síntesis el catalizador se secó a $150{ }^{\circ} \mathrm{C}$ durante 
12 h, lo que hizo que la interacción entre el HPW y el soporte fuera más fuerte. Como resultado, las microesferas preparadas exhibieron un rendimiento mejorado en la degradación de tintes bajo irradiación con UV en comparación con el HPW puro. M. Masteri-Farahani y col [103] prepararon catalizadores con dos HPA, ácido tungstofosfórico $\mathrm{y}$ fosfomolibdico (HMoW) mediante la modificación de la superficie de sílice con trimetoxi silano de aminopropilo (APTS) que produce aminopropilo. Estos materiales presentaron una alta transferencia de protones desde los HPA a los grupos amina que genera una fuerte interacción entre ellos debido a una compensación de carga que permite la inmovilización de los HPA.

También se han inmovilizado HPA con estructuras más complejas que las de tipo Keggin, como lo son los heteropoliácidos tipo Wells-Dawson. Mohammad Ghanbari y col [104] prepararon un híbrido conformado por $\mathrm{H}_{6} \mathrm{P}_{2} \mathrm{~W}_{18} \mathrm{O}_{62}$ soportado sobre $\mathrm{Fe}_{3} \mathrm{O}_{4} @ \mathrm{SiO}_{2}$ funcionalizado con 2,4-bis(3,5-dimetilpirazol)-triazina (ADMPT) y lo utilizaron como un catalizador verde, eficiente, ecológico y altamente reciclable para la síntesis eficiente de 1,4-dihidopiridinas, logrando buenos a excelentes rendimientos y en un corto tiempo. Reza Tayebee y col[105] obtuvieron catalizadores de mayor complejidad a base de heteropoliacidos de estructura Wells-Dawson, como puede verse en la Figura 9 y se evaluó en la síntesis multicomponente y en ausencia de solvente de diversos 1-aminoalquil-2-naftoles sustituidos de la reacción de $\beta$-naftol, un aldehído y benzamida con rendimientos de buenos a excelentes (4794\%) y en un corto lapso de tiempo (25-60 min).

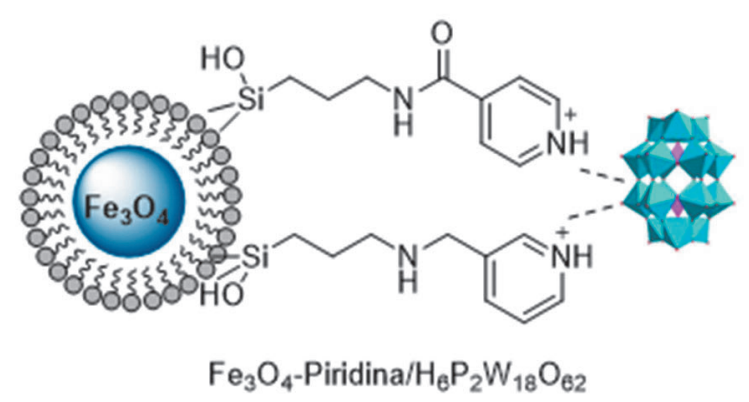

Figura 9. Esquema del catalizador $\mathrm{Fe}_{3} \mathrm{O}_{4} @ \mathrm{SiO}_{2}$ funcionalizado con piridina para soportar HPAs tipo Wells Dawson.
En nuestro grupo de investigación se sintetizaron catalizadores magnéticos basados en HPW inmovilizado en sílice mesoporosa depositada sobre partículas de magnetita $\left(\mathrm{Fe} 3 \mathrm{O} 4 @ \mathrm{SiO}_{2}\right.$ ). En el proceso de síntesis se formaron dos capas de sílice, la primera mediante hidrólisis básica del TEOS para proteger las NPMs y la segunda capa por hidrólisis ácida catalizada por el mismo HPW de tal forma que quedara inmovilizado en medio de esta capa. El catalizador presentó rendimientos del $90 \%$ en la reacción de esterificación del ácido levulínico con n-butanol para obtener levulinato de n-butilo, un subproducto de biomasa interesante por sus aplicaciones como aditivo en biocombustibles. Se estudió su cinética y se evaluaron procesos de transferencia de masa externa así como de difusión intra-partícula. Los resultados aún no están publicados.

\section{CONCLUSIONES Y PERSPECTIVAS}

El campo de la catálisis heterogénea de NPMs de óxidos de hierro es amplio, debido a las múltiples posibilidades de combinar compuestos de recubrimiento y funcionalización con fases activas. Aunque se ha avanzado bastante en la síntesis de NPMs de alta calidad, tamaños y formas controladas, aún hay bastantes retos por afrontar como por ejemplo: comprender detalladamente los mecanismos de síntesis y los fenómenos que ocurren en la escala de los nanómetros; rediseñar los métodos de síntesis existentes que a menudo requieren el uso de precursores tóxicos $\mathrm{y} / \mathrm{o}$ costosos, simplificar los procesos y lograr cada vez condiciones de reacción más suaves; realizar aportes a la ciencia de los materiales a través del avance en las técnicas de caracterización que permitan correlacionar la naturaleza química del recubrimiento y la fase activa con las propiedades magnéticas y la estabilidad de los catalizadores. Pero sobre todo, lo más importante es lograr métodos que sean reproducibles a escala industrial y ampliar sus aplicaciones tanto en Química Fina y Síntesis Orgánica como en fotocatálisis y remediación ambiental, ya que los catalizadores son herramientas indispensables para la industria química, sólo si sus aplicaciones son prácticas. En otras palabras, puede ser una tecnología sostenible clave solamente si se demuestra su utilidad en la sociedad mediante las mejoras del bienestar humano y del medio ambiente. 


\section{BIBLIOGRAFÍA}

1. Tudu B, Tiwari A (2017) Recent Developments in Perpendicular Magnetic Anisotropy Thin Films for Data Storage Applications. Vacuum 146:329-341.https:// doi.org/10.1016/j.vacuum.2017.01.031

2. Kurlyandskaya GV, Novoselova IP, Schupletsova VV, et al (2017) Nanoparticles for magnetic biosensing systems. J Magn Magn Mater 431:249-254. https://doi. org/10.1016/j.jmmm.2016.07.056

3. Yusuf SM (2012) Functional Magnetic Materials: Fundamental and Technological Aspects. In: Functional Materials. Elsevier, pp 111-154

4. NerimetlaR,PremaratneG,LiuH,KrishnanS (2018) Improved electrocatalytic metabolite production and drug biosensing by human liver microsomes immobilized on aminefunctionalized magnetic nanoparticles. Electrochim Acta 280:101-107. https://doi. org/10.1016/j.electacta.2018.05.085

5. Tan KA, Morad N, Teng TT, et al (2012) Removal of Cationic Dye by Magnetic Nanoparticle (Fe3O4) Impregnated onto Activated Maize Cob Powder and Kinetic Study of Dye Waste Adsorption. APCBEE Procedia 1:83-89. https://doi.org/10.1016/j. apcbee.2012.03.015

6. Tang SCN, Lo IMC (2013) Magnetic nanoparticles: Essential factors for sustainable environmental applications. Water Res 47:2613-2632. https://doi. org/10.1016/j.watres.2013.02.039

7. Carmona-Carmona AJ, Palomino-Ovando MA, Hernández-Cristobal O, et al (2017) Synthesis and characterization of magnetic opal $/ \mathrm{Fe}_{3} \mathrm{O}_{4}$ colloidal crystal. J Cryst Growth 462:6-11. https://doi.org/10.1016/j. jcrysgro.2016.12.105

8. Song S, Yang H, Rao R, et al (2010) High catalytic activity and selectivity for hydroxylation of benzene to phenol over multi-walled carbon nanotubes supported $\mathrm{Fe}_{3} \mathrm{O}_{4}$ catalyst. Appl Catal A Gen
375:265-271. https://doi.org/10.1016/j. apcata.2010.01.008

9. Rafiee E, Khodayari M (2016) Starch as a green source for $\mathrm{Fe}_{3} \mathrm{O}_{4} @$ carbon coreshell nanoparticles synthesis: A support for 12-tungstophosphoric acid, synthesis, characterization, and application as an efficient catalyst. Res Chem Intermed 42:3523-3536. https://doi.org/10.1007/ s11164-015-2229-5

10. Zhang Z, Zhang F, Zhu Q, et al (2011) Magnetically separable polyoxometalate catalyst for the oxidation of dibenzothiophene with $\mathrm{H}_{2} \mathrm{O}_{2}$. J Colloid Interface Sci 360:189-194. https://doi. org/10.1016/j.jcis.2011.04.045

11. Eshghi H, Khojastehnezhad A, Moeinpour F, et al (2014) Synthesis, characterization and first application of keggin-type heteropoly acids supported on silica coated $\mathrm{NiFe}_{2} \mathrm{O}_{4}$ as novel magnetically catalysts for the synthesis of tetrahydropyridines. RSC Adv 4:39782. https://doi.org/10.1039/ C4RA05133E

12. Khosroshahi ME, Ghazanfari L (2010) Preparation and characterization of silicacoated iron-oxide bionanoparticles under $\mathrm{N}_{2}$ gas. Phys E Low-Dimensional Syst Nanostructures 42:1824-1829. https://doi. org/10.1016/j.physe.2010.01.042

13. Sohn BH, Cohen RE (1997) Processible Optically Transparent Block Copolymer Films Containing Superparamagnetic Iron Oxide Nanoclusters. Chem Mater 9:264269. https://doi.org/10.1021/cm960339d

14. Robinson PJ, Dunnill P, Lilly MD (1973) The properties of magnetic supports in relation to immobilized enzyme reactors. Biotechnol Bioeng 15:603-606. https://doi. org/10.1002/bit.260150318

15. Whitesides GM, Hill CL, Brunie JC (1976) Magnetic Filtration of Small Heterogeneous Catalyst Particles. Preparation of Ferrimagnetic Catalyst Supports. Ind Eng Chem Process Des Dev 15:226-227. https:// doi.org/10.1021/i260057a040 
16. Maleki B, Eshghi H, Khojastehnezhad A, et al (2015) Silica coated magnetic $\mathrm{NiFe}_{2} \mathrm{O}_{4}$ nanoparticle supported phosphomolybdic acid; Synthesis, preparation and its application as a heterogeneous and recyclable catalyst for the one-pot synthesis of tri- and tetra-substituted imidazoles unde. RSC Adv 5:64850-64857. https:// doi.org/10.1039/C5RA10534J

17. Dadwal A, Joy PA (2018) Influence of chain length of long-chain fatty acid surfactant on the thermal conductivity of magnetite nanofluids in a magnetic field. Colloids Surfaces A Physicochem Eng Asp 555:525-531. https://doi.org/10.1016/j. colsurfa.2018.07.034

18. Du GH, Liu ZL, Xia X, et al (2006) Characterization and application of $\mathrm{Fe}_{3} \mathrm{O}_{4} /$ $\mathrm{SiO}_{2}$ nanocomposites. J Sol-Gel Sci Technol 39:285-291. https://doi.org/10.1007/ s10971-006-7780-5

19. Guo W, Wang G, Wang Q, et al (2013) Journal of Molecular Catalysis A : Chemical A hierarchical $\mathrm{Fe}_{3} \mathrm{O}_{4} @ \mathrm{P}_{4} \mathrm{VP} @ \mathrm{MoO}_{2}(\mathrm{acac})_{2}$ nanocomposite: Controlled synthesis and green catalytic application. "Journal Mol Catal A, Chem 378:344-349. https://doi. org/10.1016/j.molcata.2013.04.019

20. Sadeghzadeh SM (2015) A heteropolyacidbased ionic liquid immobilized onto $\mathrm{Fe}_{3} \mathrm{O}_{4} /$ $\mathrm{SiO}_{2} / \mathrm{Salen} / \mathrm{Mn}$ as an environmentally friendly catalyst for synthesis of cyclic carbonate. Res Chem Intermed. https://doi. org/10.1007/s11164-015-2151-x

21. Warner CL, Carter TG, Wiacek RJ, Fryxell GE (2007) Removal of Heavy Metals from Aqueous Systems with Thiol Functionalized Superparamagnetic Nanoparticles. 41:5114-5119

22. Chi Y, Yuan Q, Li Y, et al (2012) Journal of Colloid and Interface Science Synthesis of $\mathrm{Fe}_{3} \mathrm{O}_{4} @ \mathrm{SiO}_{2}-\mathrm{Ag}$ magnetic nanocomposite based on small-sized and highly dispersed silver nanoparticles for catalytic reduction of 4-nitrophenol. J Colloid Interface Sci 383:96-102. https://doi.org/10.1016/j. jcis.2012.06.027
23. Amin R, Khorshidi A, Shojaei AF, et al (2018) Immobilization of laccase on modified $\mathrm{Fe}_{3} \mathrm{O}_{4} @ \mathrm{SiO}_{2} @$ Kit-6 magnetite nanoparticles for enhanced delignification of olive pomace bio-waste. Int $\mathrm{J}$ Biol Macromol 114:106-113. https://doi. org/10.1016/j.ijbiomac.2018.03.086

24. Martínez JJ, Nope E, Rojas H, et al (2014) Dehydration of xylose to furfural and its valorization via different multicomponent reactions using sulfonated silica with magnetic properties as recyclable catalyst. Catal Letters 144:1322-1331. https://doi. org/10.1007/s10562-014-1267-8

25. Lim CW, Lee IS (2010) Magnetically recyclable nanocatalyst systems for the organic reactions. Nano Today 5:412-434. https://doi.org/10.1016/j. nantod.2010.08.008

26. Baig RBN, Nadagouda MN, Varma RS (2015) Magnetically retrievable catalysts for asymmetric synthesis. Coord Chem Rev 287:137-156. https://doi.org/10.1016/j. ccr.2014.12.017

27. Mathew DS, Juang RS (2007) An overview of the structure and magnetism of spinel ferrite nanoparticles and their synthesis in microemulsions. Chem Eng J 129:51-65. https://doi.org/10.1016/j.cej.2006.11.001

28. Weiss P (1907) L'hypothèse du champ moléculaire et la propriété ferromagnétique. J Phys Théorique Appliquée 6:661-690. https://doi.org/10.1051/ jphystap:019070060066100

29. Sorensen CM (2001) Magnetism. In: Klabunde KJ (ed) Nanoscale Materials in Chemistry. John Wiley \& Sons, Inc., New York, USA, p 169

30. Paradela RP (2013) Preparación y caracterización de nanopartículas magnéticas biocompatible. Universidad de Coruña

31. Castellanos MJR Procesos de imanación en la nanoescala mediante microscopía de 
fuerzas magnéticas. Universidad Autonoma de Madrid

32. Jahagirdar AA, Dhananjaya N, Monika DL, et al (2013) Structural, EPR, optical and magnetic properties of $\alpha-\mathrm{Fe}_{2} \mathrm{O}_{3}$ nanoparticles. Spectrochim Acta - Part A Mol Biomol Spectrosc 104:512-518. https://doi.org/10.1016/j.saa.2012.09.069

33. Wells AF (1978) Química inorgánica estructural. Reverté, Barcelona

34. Gupta AK, Naregalkar RR, Vaidya VD, Gupta M (2007) Recent advances on surface engineering of magnetic iron oxide nanoparticles and their biomedical applications. Nanomedicine 2:23-39. https://doi.org/10.2217/17435889.2.1.23

35. $\mathrm{Wu} \mathrm{W}, \mathrm{Wu} \mathrm{Z}, \mathrm{Yu} \mathrm{T}$, et al (2015) Recent progress on magnetic iron oxide nanoparticles: synthesis, surface functional strategies and biomedical applications. Sci Technol Adv Mater 16:023501. https://doi. org/10.1088/1468-6996/16/2/023501

36. Bandhu A, Mukherjee S, Acharya S, et al (2009) Dynamic magnetic behaviour and M??ssbauer effect measurements of magnetite nanoparticles prepared by a new technique in the co-precipitation method. Solid State Commun 149:1790-1794. https://doi.org/10.1016/j.ssc.2009.07.018

37. Faraji M, Yamini Y, Rezaee M (2010) Magnetic Nanoparticles: Synthesis, Stabilization, Functionalization, Characterization, and Applications. Iran Chem Soc 7:1-37

38. Li X, Xu G, Liu Y, He T (2011) Magnetic $\mathrm{Fe}_{3} \mathrm{O}_{4}$ Nanoparticles: Synthesis and Application in Water Treatment. 14-24

39. Majewski P, Thierry B (2007) Functionalized Magnetite Nanoparticles Synthesis,Properties, and BioApplications. Crit Rev Solid State Mater Sci 32:203-215. https://doi. org/10.1080/10408430701776680
40. Perez De Berti IO, Cagnoli M V, Pecchi G, et al (2013) Alternative low-cost approach to the synthesis of magnetic iron oxide nanoparticles by thermal decomposition of organic precursors. Nanotechnology 24:175601. https://doi.org/10.1088/0957$4484 / 24 / 17 / 175601$

41. Babes L, Jacques J, Jeune L, Jallet P (1999) Synthesis of Iron Oxide Nanoparticles Used as MRI Contrast Agents: A Parametric Study. J Colloid Interface Sci 212:474-482

42. Faiyas APA, Vinod EM, Joseph J, et al (2010) Dependence of $\mathrm{pH}$ and surfactant effect in the synthesis of magnetite $\left(\mathrm{Fe}_{3} \mathrm{O}_{4}\right)$ nanoparticles and its properties. J Magn Magn Mater 322:400-404. https://doi. org/10.1016/j.jmmm.2009.09.064

43. Shen YF, Tang J, Nie ZH, et al (2009) Preparation and application of magnetic $\mathrm{Fe} 3 \mathrm{O} 4$ nanoparticles for wastewater purification. Sep Purif Technol 68:312-319. https://doi.org/10.1016/j. seppur.2009.05.020

44. Bui TQ, Thi H, Ngo M, Tran HT (2018) Journal of Science: Advanced Materials and Devices Surface-protective assistance of ultrasound in synthesis of superparamagnetic magnetite nanoparticles and in preparation of. J Sci Adv Mater Devices 3:323-330. https://doi. org/10.1016/j.jsamd.2018.07.002

45. Vaidya S, Science N (2018) Microemulsion Methods for Synthesis of Nanostructured Materials $\square$. Elsevier Ltd.

46. Chen AZ, Wu C, Zhang Z, Chen Z (2018) Synthesis, functionalization, and nanomedical applications of functional magnetic nanoparticles. Chinese Chem Lett. https://doi.org/10.1016/j.cclet.2018.08.007

47. Tan TTY, Liu S, Zhang Y, et al (2011) Microemulsion Preparative Methods (Overview). In: Comprehensive Nanoscience and Technology. Elsevier, pp 399-441 
48. Zhou ZH, Wang J, Liu X, Chan HSO (2001) Synthesis of $\mathrm{Fe}_{3} \mathrm{O}_{4}$ nanoparticles from emulsions. https://doi.org/10.1039/ b100758k

49. Bumajdad A, Eastoe J, Zaki MI, et al (2007) Generation of metal oxide nanoparticles in optimised microemulsions. J Colloid Interface Sci 312:68-75. https://doi. org/10.1016/j.jcis.2006.09.007

50. Feng S, Xu R (2001) New Materials in Hydrothermal Synthesis. Acc Chem Res 34:239-247. https://doi.org/10.1021/ ar0000105

51. Nemati A, Shadpour S, Khalafbeygi H, Barkhi M (2014) Hydrothermal Synthesis and Size Control of $\mathrm{Fe}_{3} \mathrm{O}_{4}$ Nanoparticles in the Presence of 2,2',2",2"'-(ethane-1,2diylbis(azanetriyl))tetraacetohydrazide. Synth React Inorganic, Met Nano-Metal Chem 44:1161-1165. https://doi.org/10.10 80/15533174.2013.797443

52. Fu R, Wu X, Wang X, et al (2018) Lowtemperature hydrothermal fabrication of $\mathrm{Fe}_{3} \mathrm{O}_{4}$ nanostructured solar selective absorption films. Appl Surf Sci 458:629-637. https://doi.org/10.1016/j. apsusc. 2018.07 .063

53. Jiao Y, Wan C, Bao W, et al (2018) Facile hydrothermal synthesis of $\mathrm{Fe}_{3} \mathrm{O}_{4} @$ cellulose aerogel nanocomposite and its application in Fenton-like degradation of Rhodamine B. Carbohydr Polym 189:371-378. https:// doi.org/10.1016/j.carbpol.2018.02.028

54. Hernández-Hernández AA, ÁlvarezRomero GA, Castañeda-Ovando A, et al (2018) Optimization of microwavesolvothermal synthesis of $\mathrm{Fe}_{3} \mathrm{O}_{4}$ nanoparticles. Coating, modification, and characterization. Mater Chem Phys 205:113-119. https://doi.org/10.1016/j. matchemphys.2017.11.009

55. Deng H, Li X, Peng Q, et al (2005) Monodisperse Magnetic Single-Crystal Ferrite Microspheres. Angew Chemie Int Ed 44:2782-2785. https://doi.org/10.1002/ anie. 200462551
56. Li C, Wei Y, Liivat A, et al (2013) Microwave-solvothermal synthesis of $\mathrm{Fe} 3 \mathrm{O} 4$ magnetic nanoparticles. Mater Lett 107:23-26. https://doi.org/10.1016/j. matlet.2013.05.117

57. Laurent S, Forge D, Port M, et al (2008) Magnetic Iron Oxide Nanoparticles : Synthesis, Stabilization, Vectorization , Physicochemical Characterizations, and Biological Applications. 2064-2110

58. Wang D, Astruc D (2014) Fast-Growing Field of Magnetically Recyclable Nanocatalysts. https://doi.org/10.1021/ cr500134h

59. Zhang P, Wang B, Williams GR, et al (2013) Self-assembled core-shell $\mathrm{Fe}_{3} \mathrm{O}_{4} @$ $\mathrm{SiO}_{2}$ nanoparticles from electrospun fibers. Mater Res Bull 48:3058-3064. https://doi. org/10.1016/j.materresbull.2013.04.052

60. Stöber W, Fink A, Bohn E (1968) Controlled growth of monodisperse silica spheres in the micron size range. J Colloid Interface Sci 26:62-69. https://doi.org/10.1016/00219797(68)90272-5

61. Chae SS, Hwang BH, Jang WS, et al (2012) Homogeneous liquid crystal alignment on inorganic-organic hybrid silica thin films derived by the sol-gel method. Soft Matter 8:1437. https://doi.org/10.1039/ c1sm06592k

62. Goksu EI, Hoopes MI, Nellis BA, et al (2010) Silica xerogel/aerogel-supported lipid bilayers: Consequences of surface corrugation. Biochim Biophys Acta Biomembr 1798:719-729. https://doi. org/10.1016/j.bbamem.2009.09.007

63. Mariela A. Agotegaray VLL (2017) Microemulsion method. In: Silica-coated Magnetic Nanoparticles: An Insight into Targeted Drug Delivery and Toxicology. SpringerBriefs, $\mathrm{p} 42$

64. DING H, ZHAO Y, DUAN Q, et al (2017) Efficient removal of phosphate from aqueous solution using novel magnetic 
nanocomposites with $\mathrm{Fe}_{3} \mathrm{O}_{4} @ \mathrm{SiO}_{2}$ core and mesoporous $\mathrm{CeO} 2$ shell. J Rare Earths 35:984-994. https://doi.org/10.1016/ S1002-0721(17)61003-2

65. Lai L, Xie Q, Chi L, et al (2016) Adsorption of phosphate from water by easily separable Fe3O4@SiO2core/shell_magnetic nanoparticles functionalized with hydrous lanthanum oxide. J Colloid Interface Sci 465:76-82. $\quad$ https://doi.org/10.1016/j. jcis.2015.11.043

66. Ghotbinejad M, Khosropour AR, Mohammadpoor-Baltork I, et al (2014) Ultrasound-assisted $\mathrm{C}-\mathrm{C}$ coupling reactions catalyzed by unique SPION-A-Pd(EDTA) as a robust nanocatalyst. RSC Adv 4:8590. https://doi.org/10.1039/c3ra45790g

67. Wang D, Salmon L, Ruiz J, Astruc D (2013) A recyclable ruthenium(ii) complex supported on magnetic nanoparticles: a regioselective catalyst for alkyne-azide cycloaddition. Chem Commun 49:6956. https://doi.org/10.1039/c3cc43048k

68. Heidari F, Hekmati M, Veisi H (2017) Magnetically separable and recyclable $\mathrm{Fe}_{3} \mathrm{O}_{4} @ \mathrm{SiO}_{2} /$ isoniazide/Pd nanocatalyst for highly efficient synthesis of biaryls by Suzuki coupling reactions. J Colloid Interface Sci 501:175-184. https://doi. org/10.1016/j.jcis.2017.04.054

69. Tzounis L, Logothetidis $\mathrm{S}$ (2017) $\mathrm{Fe}_{3} \mathrm{O}_{4} @$ $\mathrm{SiO}_{2}$ core shell particles as platforms for the decoration of $\mathrm{Ag}$ nanoparticles. Mater Today Proc 4:7076-7082. https://doi. org/10.1016/j.matpr.2017.07.041

70. Esmaeilpour M, Sardarian AR, Javidi J (2012) Schiff base complex of metal ions supported on superparamagnetic $\mathrm{Fe}_{3} \mathrm{O}_{4} @$ $\mathrm{SiO}_{2}$ nanoparticles: An efficient, selective and recyclable catalyst for synthesis of 1,1-diacetates from aldehydes under solvent-free conditions. Appl Catal A Gen 445-446:359-367. https://doi. org/10.1016/j.apcata.2012.09.010

71. Esmaeilpour M, Sardarian AR, Javidi J (2014) Synthesis and characterization of
Schiff base complex of $\mathrm{Pd}(\mathrm{II})$ supported on superparamagnetic $\mathrm{Fe}_{3} \mathrm{O}_{4} @ \mathrm{SiO}_{2}$ nanoparticles and its application as an efficient copper- and phosphine ligandfree recyclable catalyst for SonogashiraHagihara coupling reactions. J Organomet Chem 749:233-240. https://doi. org/10.1016/j.jorganchem.2013.10.011

72. Wan H, Wu Z, Chen W, et al (2015) Heterogenization of ionic liquid based on mesoporous material as magnetically recyclable catalyst for biodiesel production. J Mol Catal A Chem 398:127-132. https:// doi.org/10.1016/j.molcata.2014.12.002

73. Kim E-J, Lee C-S, Chang Y-Y, Chang Y-S (2013)Hierarchically Structured Manganese Oxide-Coated Magnetic Nanocomposites for the Efficient Removal of Heavy Metal Ions from Aqueous Systems. ACS Appl Mater Interfaces 5:9628-9634. https://doi. org/10.1021/am402615m

74. Zhao Z, Geng C, Yang C, et al (2018) Anovel flake-ball-like magnetic $\mathrm{Fe}_{3} \mathrm{O}_{4} / \gamma-\mathrm{MnO}_{2}$ meso-porous nano-composite: Adsorption of fluorinion and effect of water chemistry. Chemosphere 209:173-181. https://doi. org/10.1016/j.chemosphere.2018.06.104

75. Yang Q, Song H, Li Y, et al (2017) Flowerlike core-shell $\mathrm{Fe}_{3} \mathrm{O}_{4} @ \mathrm{MnO}_{2}$ microspheres: Synthesis and selective removal of Congo red dye from aqueous solution. J Mol Liq 234:18-23. https://doi.org/10.1016/j. molliq.2017.03.028

76. Tanabe K (2003) Catalytic application of niobium compounds. Catal Today 78:65-77. https://doi.org/10.1016/S09205861(02)00343-7

77. Opris C,CojocaruB, GheorgheN, etal(2016) Lignin fragmentation over magnetically recyclable composite $\mathrm{Co} @ \mathrm{Nb}_{2} \mathrm{O}_{5} @ \mathrm{Fe}_{3} \mathrm{O}_{4}$ catalysts. J Catal 339:209-227. https://doi. org/10.1016/j.jcat.2016.04.002

78. Liu J, Sun B, Hu J, et al (2010) Aqueousphase reforming of ethylene glycol to hydrogen on $\mathrm{Pd} / \mathrm{Fe}_{3} \mathrm{O}_{4}$ catalyst prepared by 
co-precipitation: Metal-support interaction and excellent intrinsic activity. J Catal 274:287-295. https://doi.org/10.1016/j. jcat.2010.07.014

79. Zhang R, Liu J, Li F, et al (2011) Magnetically Separable and Versatile $\mathrm{Pd} / \mathrm{Fe} 3 \mathrm{O} 4$ Catalyst for Efficient Suzuki Cross-Coupling Reaction and Selective Hydrogenation of Nitroarenes. Chinese $\mathrm{J}$ Chem 29:525-530. https://doi.org/10.1002/ cjoc. 201190117

80. Shang J, Guo X, Shi F, et al (2011) $\mathrm{N}$-substituted carbamates syntheses with alkyl carbamates as carbonyl source over Ni-promoted $\mathrm{Fe}_{3} \mathrm{O}_{4}$ catalyst. J Catal 279:328-336. https://doi.org/10.1016/j. jcat.2011.01.030

81. Yang B, Zhang Q, Ma X, et al (2016) Preparation of a magnetically recoverable nanocatalyst via cobalt-doped $\mathrm{Fe}_{3} \mathrm{O}_{4}$ nanoparticles and its application in the hydrogenation of nitroarenes. Nano Res 9:1879-1890. https://doi.org/10.1007/ s12274-016-1080-3

82. Feyzi M, Hassankhani A, Rafiee HR (2013) Preparation and characterization of $\mathrm{Cs} / \mathrm{Al} / \mathrm{Fe}_{3} \mathrm{O}_{4}$ nanocatalysts for biodiesel production. Energy Convers Manag 71:62-68. https://doi.org/10.1016/j. enconman.2013.03.022

83. Zhan J, Tian G, Jiang L, et al (2008) Superparamagnetic polyimide $/ \gamma-\mathrm{Fe}_{2} \mathrm{O}_{3}$ nanocomposite films: Preparation and characterization. 516:6315-6320. https:// doi.org/10.1016/j.tsf.2007.12.090

84. Wang L, Cole M, Li J, et al (2015) Polymer grafted recyclable magnetic nanoparticles. Polym Chem 6:248-255. https://doi. org/10.1039/C4PY01134A

85. Liu ZL, Ding ZH, Yao KL, et al (2003) Preparation and characterization of polymer-coated core - shell structured magnetic microbeads. 265:98-105. https:// doi.org/10.1016/S0304-8853(03)00230-0
86. Utkan G, Sayar F, Batat P, et al (2011) Synthesis and characterization of nanomagnetite particles and their polymer coated forms. J Colloid Interface Sci 353:372-379. https://doi.org/10.1016/j. jcis.2010.09.081

87. Liu B, Zhang W, Yang F, et al (2011) Facile Method for Synthesis of $\mathrm{Fe}_{3} \mathrm{O}_{4} @$ Polymer Microspheres and Their Application As Magnetic Support for Loading Metal Nanoparticles. J Phys Chem C 115:1587515884. https://doi.org/10.1021/jp204976y

88. Liu G, Wang H, Yang X (2009) Synthesis of $\mathrm{pH}$-sensitive hollow polymer microspheres with movable magnetic core. Polymer (Guildf) 50:2578-2586. https://doi. org/10.1016/j.polymer.2009.04.002

89. Karaca E, Şatır M, Kazan S, et al (2015) Synthesis, characterization and magnetic properties of $\mathrm{Fe}_{3} \mathrm{O}_{4}$ doped chitosan polymer. J Magn Magn Mater 373:53-59. https://doi. org/10.1016/j.jmmm.2014.02.016

90. Sinha V., Singla A., Wadhawan S, et al (2004) Chitosan microspheres as a potential carrier for drugs. Int J Pharm 274:1-33. https://doi. org/10.1016/j.ijpharm.2003.12.026

91. Naghipour A, Fakhri A (2016) Heterogeneous $\mathrm{Fe}_{3} \mathrm{O}_{4} @$ chitosan-Schiff base $\mathrm{Pd}$ nanocatalyst: Fabrication, characterization and application as highly efficient and magnetically-recoverable catalyst for Suzuki-Miyaura and HeckMizoroki C-C coupling reactions. Catal Commun 73:39-45. https://doi. org/10.1016/j.catcom.2015.10.002

92. Jonoobi M, Oladi R, Davoudpour Y, et al (2015) Different preparation methods and properties of nanostructured cellulose from various natural resources and residues: a review. Cellulose 22:935-969. https://doi. org/10.1007/s10570-015-0551-0

93. Hokkanen S, Bhatnagar A, Sillanpää M (2016) A review on modification methods to cellulose-based adsorbents to 
improve adsorption capacity. Water Res 91:156-173. https://doi.org/10.1016/j. watres.2016.01.008

94. Zarei S, Niad M, Raanaei H (2018) The removal of mercury ion pollution by using Fe3O4-nanocellulose: Synthesis, characterizations and DFT studies. J Hazard Mater 344:258-273. https://doi. org/10.1016/j.jhazmat.2017.10.009

95. Izumi Y, Matsuo K, Urabe K (1983) Efficient homogeneous acid catalysis of heteropoly acid and its characterization through ether cleavage reactions. J Mol Catal 18:299-314. https://doi.org/10.1016/ S0304-5102(83)80004-2

96. Kozhevnikov I V., Matveev KI (1983) Homogeneous catalysts based on heteropoly acids (review). Appl Catal 5:135-150. https://doi.org/10.1016/01669834(83)80128-6

97. Morin P, Hamad B, Sapaly G, et al (2007) Transesterification of rapeseed oil with ethanol. I. Catalysis with homogeneous Keggin heteropolyacids. Appl Catal A Gen 330:69-76. https://doi.org/10.1016/j. apcata.2007.07.011

98. Kamiya Y, Okuhara T, Misono M, et al (2008) Catalytic chemistry of supported heteropolyacids and their applications as solid acids to industrial processes. Catal Surv from Asia 12:101-113. https://doi. org/10.1007/s10563-008-9043-7

99. Kong A, Wang P, Zhang H, et al (2012) Applied Catalysis A: General One-pot fabrication of magnetically recoverable acid nanocatalyst, heteropolyacids/ chitosan $/ \mathrm{Fe}_{3} \mathrm{O}_{4}$, and its catalytic performance. "Applied Catal A, Gen 417418:183-189. https://doi.org/10.1016/j. apcata.2011.12.040
100. Nikbakht F, Heydari A, Saberi D, Azizi K (2013) Oxidation of secondary amines to nitrones using magnetically separable tungstophosphoric acid supported on silica-encapsulated $\gamma-\mathrm{Fe}_{2} \mathrm{O}_{3}$ nanoparticles. Tetrahedron Lett 54:6520-6523. https://doi. org/10.1016/j.tetlet.2013.09.090

101. Yuan J, Yue P, Wang L (2010) A study on the magnetically supported heteropolyacid nanophase catalysts. Powder Technol 202:190-193. https://doi.org/10.1016/j. powtec.2010.04.020

102. Zhao L, Chi Y, Yuan Q, et al (2013) Phosphotungstic acid anchored to aminofunctionalized core-shell magnetic mesoporous silica microspheres: A magnetically recoverable nanocomposite with enhanced photocatalytic activity. J Colloid Interface Sci 390:70-77. https:// doi.org/10.1016/j.jcis.2012.08.059

103. Masteri-Farahani M, Movassagh J, Taghavi F, et al (2012) Magnetite-polyoxometalate hybrid nanomaterials: Synthesis and characterization. Chem Eng J 184:342-346. https://doi.org/10.1016/j.cej.2011.12.094

104. Mohammad Ghanbari SM and MS (2018) Green Chemistry Letters and Reviews $\mathrm{Fe}_{3} \mathrm{O}_{4} @ \mathrm{SiO}_{2} @ \mathrm{ADMPT} / \mathrm{H}_{6} \mathrm{P}_{2} \mathrm{~W}_{18} \mathrm{O}_{62}: \quad \mathrm{a}$ novel Wells-Dawson heteropolyacid-based magnetic inorganic - organic nanohybrid material as potent Lewis acid catalyst for the efficient synthesis of. Green Chem Lett Rev 8253:111-124. https://doi.org/10.1080/ 17518253.2018.1445781

105. Tayebee R, Amini MM, Rostamian H, Aliakbari A (2014) Preparation and characterization of a novel Wells-Dawson heteropolyacid-based magnetic inorganicorganic nanohybrid catalyst $\mathrm{H}_{6} \mathrm{P}_{2} \mathrm{~W}_{18} \mathrm{O}_{62} /$ pyridino- $\mathrm{Fe}_{3} \mathrm{O}_{4}$ for the efficient synthesis of 1-ami. Dalt Trans 43:1550-1563. https:// doi.org/10.1039/c3dt51594j 